\title{
Associated top quark pair production with a heavy boson: differential cross sections at NLO + NNLL accuracy
}

\author{
Anna Kulesza ${ }^{1, a}$, Leszek Motyka ${ }^{2, b}$, Daniel Schwartländer ${ }^{1, c}$, Tomasz Stebel $^{2,3, d}$, Vincent Theeuwes $^{4, e}{ }_{\mathbb{D}}$ \\ ${ }^{1}$ Institute for Theoretical Physics, WWU Münster, 48149 Münster, Germany \\ 2 Institute of Physics, Jagiellonian University, S.Łojasiewicza 11, 30-348 Kraków, Poland \\ ${ }^{3}$ Institute of Nuclear Physics PAN, Radzikowskiego 152, 31-342 Kraków, Poland \\ ${ }^{4}$ Institute for Theoretical Physics, Georg-August-University Göttingen, Friedrich-Hund-Platz 1, 37077 Göttingen, Germany
}

Received: 23 January 2020 / Accepted: 28 April 2020 / Published online: 15 May 2020

(c) The Author(s) 2020

\begin{abstract}
We present theoretical predictions for selected differential cross sections for the process $p p \rightarrow t \bar{t} B$ at the LHC, where $B$ can be a Higgs $(H)$, a $Z$ or a $W$ boson. The predictions are calculated in the direct $\mathrm{QCD}$ framework up to the next-to-next-leading logarithmi accuracy and matched to the complete NLO results including QCD and electroweak effects. Additionally, results for the total cross sections are provided. The calculations deliver a significant improvement of the theoretical predictions, especially for the $t \bar{t} H$ and the $t \bar{t} Z$ production. In these cases, predictions for both the total and differential cross sections are remarkably stable with respect to the central scale choice and carry a substantially reduced scale uncertainty in comparison with the complete NLO predictions.
\end{abstract}

\section{Contents}

1 Introduction $\ldots \ldots \ldots \ldots \ldots \ldots$

2 NNLL resummation . . . . . . . . . . 3

3 Numerical results . . . . . . . . . . . . . . . . 4

3.1 Total cross sections . . . . . . . . . . . 5

3.2 Differential distributions . . . . . . . . . 7

4 Summary . . . . . . . . . . . . . 11

References . . . . . . . . . . . . . . . . 17

a e-mail: anna.kulesza@uni-muenster.de

b e-mail: leszekm@th.if.uj.edu.pl

ce-mail: d_schw20@uni-muenster.de

d e-mail: tomasz.stebel@uj.edu.pl

e e-mail: vincent.theeuwes@uni-goettingen.de (corresponding author)

\section{Introduction}

In the recent years the first two stages of the Large Hadron Collider (LHC) physics program, Run 1 and Run 2, have been completed with spectacular discovery of the Higgs boson, but with no indications of New Physics signals. In the future High Luminosity phase of the LHC, the luminosity will be increased by an order of magnitude. Much larger statistical samples and better understanding of the systematic uncertainties will lead to a substantial reduction of experimental errors in the coming Run 3 and, specifically, at the High Luminosity LHC. In particular, one expects that for many important processes the experimental errors will get significantly smaller than estimated theoretical uncertainties. This context makes it absolutely necessary to maximize the theoretical precision of the Standard Model predictions for the processes measurable at the LHC. Together with increasing experimental accuracy this results in better knowledge of the Standard Model parameters, precise determination of the Higgs boson properties, and possibly to finding deviations from the Standard Model caused by new particles or interactions.

Studies of the most massive particles of the Standard Model: the top quark, the Higgs boson and the heavy electroweak gauge bosons: $W^{ \pm}$and $Z$ are of particular interest. The Higgs boson sector is expected to exhibit enhanced sensitivity to New Physics effects since the measured value of the Higgs boson mass is highly unnatural within the Standard Model. Complementary, the Higgs boson and top sector are also crucial for solving the vital problem of the electroweak vacuum stability. The LHC energy and luminosity have allowed to search for and measure, for the first time, the processes of the associated production of the top-antitop quark pair with a heavy boson: the Higgs boson $H$ [1-7], $W^{ \pm}$or $Z$ [8-15]. These processes provide independent con- 
straints of the electroweak couplings of the top quark and of the top quark Yukawa coupling. The most recent experimental analyses of $p p \rightarrow t \bar{t} W^{ \pm}$and $p p \rightarrow t \bar{t} Z$ were performed at $\sqrt{S}=13 \mathrm{TeV}$ by the ATLAS [14] and CMS [15] collaborations, using a data sample corresponding to a fraction of the collected Run 2 luminosity. In particular, the $Z$ boson's $p_{T}$ distribution was measured for the first time very recently [15].

The current experimental accuracy $[14,15]$ is already similar to the estimated theoretical uncertainty of the next-toleading (NLO) QCD predictions for the $t \bar{t} Z$ production and will only get better in the future measurements. Over the years a great deal of effort was invested to improve the theoretical description of the $p p \rightarrow t \bar{t} H$ and $p p \rightarrow t \bar{t} V$ ( $V=W^{ \pm}, Z$ ) processes. The calculation of the NLO QCD corrections [16-30] was supplemented by their matching to partons showers [31-36]. The electroweak corrections and the combined electroweak corrections with the QCD corrections are also known [37-40]. Furthermore, NLO QCD corrections were studied for production of the $\mathrm{Z}$ boson with offshell top quarks [41], while for the Higgs boson production with off-shell top quarks NLO QCD and NLO EW were calculated $[42,43]$. The dominant theoretical uncertainty comes from the higher orders of the QCD perturbative expansion and is estimated by variation of the factorization and renormalization scales. The fixed order results may be systematically improved by applying the soft gluon resummation technique. It is a rigorous approximation scheme based on the hard factorisation theorem within the leading twist collinear approximation. The soft gluon resummation picks up the leading contributions at all orders of QCD perturbative series enhanced by powers of logarithms with arguments depending on energies of soft gluons in higher order diagrams. The resummation is a standard tool to improve theoretical precision of fixed order calculations in QCD. The resummation is typically performed in one of the two frameworks: either using the direct QCD approach $[44,45]$ or within the Soft Collinear Effectve Theory (SCET) formulation [46-49].

The practical application of the soft gluon resummation to five-leg ( $\rightarrow 3$ ) particle processes with four legs carrying colour and with multiple colour channels has been developed in recent years within the direct QCD approach [50-57] and using SCET [58-63]. In the case of the associated $t \bar{t} H$ hadroproduction, first the direct QCD framework has been applied the absolute threshold limit at the improved next-toleading logarithmic (NLL) accuracy [50]. In the following papers various approaches were developed $[51,58]$ to reach the full next-to-next-to-leading logarithmic (NNLL) accuracy for the $t \bar{t} H$ process for the resummation performed in the invariant mass threshold limit [52,59]. A closely related analysis of soft gluon effects for a simpler $2 \rightarrow 3$ process, the $t \bar{t} W^{ \pm}$hadroproduction, started with resummation at the NNLL accuracy [63]. Results at the NNLL accuracy were also obtained by other groups and calculations were extended for the associated $t \bar{t} Z$ production [54,55,57,60-62]. Thus the NNLL resummation framework for $t \bar{t} B$ hadroproduction is available for all heavy bosons $B=H, Z$ or $W^{ \pm}$. Recently, it has been also proposed to improve the NLL treatment of the $t \bar{t} H$ cross section by resummation of the QCD Coulomb corrections in the absolute threshold resummation scheme [64].

The direct QCD and SCET frameworks are formally equivalent at a given logarithmic accuracy, but subleading terms beyond the formal accuracy may differ, depending for instance on the scale setting procedures. It is therefore important to compare the results obtained within both approaches in order to provide the highest level of theoretical reliability and to better understand theoretical uncertainties. So far the total cross sections for $t \bar{t} B$ hadroproduction at the LHC and the $t \bar{t} B$ invariant mass distributions were obtained through the NLO QCD + NNLL accuracy in both frameworks. In addition, using the SCET procedure a set of other differential distributions was calculated [58-61]. In the direct QCD approach, besides the invariant mass distribution of $t \bar{t} B$ $[52,55]$ only the $p_{T}$ distribution of the $Z$ boson was presented [57] to date. In this paper we fill this gap by providing the $\mathrm{NLO}+\mathrm{NNLL}$ predictions of various kinematic distributions that are or may be measured in the $t \bar{t} B$ hadroproduction at the LHC. Moreover we calculate several new distributions, that have not been considered in the literature yet. These are rapidity and azimuthal angle difference distributions of different pairs of the final state particles. In all calculations presented here we include also electroweak effects up to the NLO accuracy. Calculations at NLO (QCD + EW $)+$ NNLL accuracy in the direct QCD framework were first performed for the $p_{T}$ distribution of the $Z$ boson in $t \bar{t} Z$ hadroproduction [57], and then for a set of differential $t \bar{t} B$ distributions in the SCET approach [62]. Also in the context of including electroweak effects this paper extends the results of [57] using the same approach in the direct QCD framework. Thus, the main goal of this analysis is to fully use the developed theoretical framework to provide the most accurate theory predictions for differential cross sections relevant for experimental measurements. Special attention is given to the dependencies on various choices of central scales, and we show that the soft gluon resummation leads to high stability against the scale variations for all studied distributions.

The paper is organized as follows: in Sect. 2 a short overview of the applied theoretical framework is given, in Sect. 3 the total and differential cross sections through NLO $(\mathrm{QCD}+\mathrm{EW})+\mathrm{NNLL}$ accuracy are presented for $p p \rightarrow t \bar{t} H$, $p p \rightarrow t \bar{t} W^{ \pm}$and $p p \rightarrow t \bar{t} Z$, and conclusions are given in Sect. 4. 


\section{NNLL resummation}

In the following, we use the formalism of threshold resummation in the invariant mass threshold limit, $Q^{2} / \hat{s} \rightarrow 1$, where $Q^{2}=\left(p_{t}+p_{\bar{t}}+p_{B}\right)^{2}$ and $\hat{s}$ is the invariant mass squared of the colliding parton pair. Since the formalism has been developed and applied to the $p p \rightarrow t \bar{t} B$ process by us in the past [51-56], here we only present the relevant key equations and refer the reader to our earlier work for a detailed description.

Resummation techniques allow to systematically take into account logarithmic terms of the type $\alpha_{\mathrm{s}}^{n}\left[\log ^{r}(1-z) /\right.$ $(1-z)]_{+}$, with $r \leq 2 n-1$ and $z=Q^{2} / \hat{s}$, up to all orders in $\alpha_{\mathrm{s}}$. The resummation is performed in Mellin space, where Mellin moments are taken with respect to the hadronic threshold $\rho=Q^{2} / S$. The Mellin transform turns the logarithms with arguments expressed in terms of the $z$ variable into logarithms of the Mellin moment $N$. The systematic inclusion of the logarithmic terms is performed through means of the factorized expression for the partonic differential cross section,

$$
\begin{aligned}
& \frac{d \tilde{\hat{\sigma}}_{i j \rightarrow k l B}^{(\mathrm{NNLL})}}{d Q^{2}}\left(N, Q^{2},\left\{m^{2}\right\}, \mu_{\mathrm{F}}^{2}, \mu_{\mathrm{R}}^{2}\right) \\
& =\int d \Phi_{3} \operatorname{Tr}\left[\mathbf{H}\left(Q^{2}, \Phi_{3},\left\{m^{2}\right\}, \mu_{\mathrm{F}}^{2}, \mu_{\mathrm{R}}^{2}\right)\right. \\
& \left.\quad \mathbf{S}\left(N+1, Q^{2}, \Phi_{3},\left\{m^{2}\right\}, \mu_{\mathrm{R}}^{2}\right)\right] \\
& \quad \times \Delta^{i}\left(N+1, Q^{2}, \mu_{\mathrm{F}}^{2}, \mu_{\mathrm{R}}^{2}\right) \Delta^{j}\left(N+1, Q^{2}, \mu_{\mathrm{F}}^{2}, \mu_{\mathrm{R}}^{2}\right),
\end{aligned}
$$

where the functions $\mathbf{H}, \mathbf{S}, \Delta$ are the hard, soft and initial state radiation factors, correspondingly. The first two, $\mathbf{H}$ and $\mathbf{S}$, are matrices in colour space and depend on the three particle phase space, $\Phi_{3}$. The function $\mathbf{H}$ contains information on the hard-off shell dynamics and includes LO contributions as well as virtual corrections split into colour channels. The soft function, $\mathbf{S}$, describes the soft-wide angle emissions and is given by a solution of the relevant renormalization group equation. The initial state emissions, $\Delta^{i} \Delta^{j}$ take into account soft/collinear emissions from the initial state partons $i$ and $j$. The cross sections depend on the renormalisation scale $\mu_{\mathrm{R}}$, the factorisation scale $\mu_{\mathrm{F}}$, and the masses of particles (squared) $\left\{m^{2}\right\}$.

The inclusive total cross section is computed by integrating the expression over $Q^{2}$. For the differential distributions of an observable $\mathcal{O}$, in addition to the integration over $Q^{2}$, a function $\mathcal{F}_{\mathcal{O}}$ is introduced which includes a phase space restriction defining the observable $\mathcal{O}$ at the hand of a delta function:

$$
\frac{d \tilde{\hat{\sigma}}_{i j \rightarrow k l B}^{(\mathrm{NNLL})}}{d \mathcal{O}}\left(N, \mathcal{O},\left\{m^{2}\right\}, \mu_{\mathrm{F}}^{2}, \mu_{\mathrm{R}}^{2}\right)
$$

$$
\begin{aligned}
= & \int d Q^{2} \int d \Phi_{3} \operatorname{Tr}\left[\mathbf{H}\left(Q^{2}, \Phi_{3},\left\{m^{2}\right\}, \mu_{\mathrm{F}}^{2}, \mu_{\mathrm{R}}^{2}\right)\right. \\
& \left.\mathbf{S}\left(N+1, Q^{2}, \Phi_{3},\left\{m^{2}\right\}, \mu_{\mathrm{R}}^{2}\right)\right] \\
& \times \Delta^{i}\left(N+1, Q^{2}, \mu_{\mathrm{F}}^{2}, \mu_{\mathrm{R}}^{2}\right) \Delta^{j}(N \\
& \left.+1, Q^{2}, \mu_{\mathrm{F}}^{2}, \mu_{\mathrm{R}}^{2}\right) \mathcal{F}_{\mathcal{O}}\left(Q^{2}, \Phi_{3},\left\{m^{2}\right\}\right) .
\end{aligned}
$$

The resummed hadronic $h_{1} h_{2}$ cross sections of different accuracy denoted by "res" in the following are matched with the full NLO cross section according to

$$
\begin{aligned}
& \frac{d \sigma_{h_{1} h_{2} \rightarrow k l B}^{(\text {matched }}}{d \mathcal{O}}\left(\mathcal{O},\left\{m^{2}\right\}, \mu_{\mathrm{F}}^{2}, \mu_{\mathrm{R}}^{2}\right) \\
& =\frac{d \sigma_{h_{1} h_{2} \rightarrow k l B}^{(\mathrm{NLO})}}{d \mathcal{O}}\left(\mathcal{O},\left\{m^{2}\right\}, \mu_{\mathrm{F}}^{2}, \mu_{\mathrm{R}}^{2}\right) \\
& \quad+\frac{d \sigma_{h_{1} h_{2} \rightarrow k l B}^{(\mathrm{res}-\exp )}}{d \mathcal{O}}\left(\mathcal{O},\left\{m^{2}\right\}, \mu_{\mathrm{F}}^{2}, \mu_{\mathrm{R}}^{2}\right)
\end{aligned}
$$

with

$$
\begin{aligned}
& \frac{d \sigma_{h_{1} h_{2} \rightarrow k l B}^{(\text {res }-\exp )}}{d \mathcal{O}}\left(\mathcal{O},\left\{m^{2}\right\}, \mu_{\mathrm{F}}^{2}, \mu_{\mathrm{R}}^{2}\right) \\
& =\sum_{i, j} \int_{\mathrm{C}} \frac{d N}{2 \pi i} \rho^{-N} f_{i / h_{1}}^{(N+1)}\left(\mu_{\mathrm{F}}^{2}\right) f_{j / h_{2}}^{(N+1)}\left(\mu_{\mathrm{F}}^{2}\right) \\
& \quad \times\left[\frac{d \tilde{\hat{\sigma}}_{i j \rightarrow k l B}^{(\mathrm{res})}}{d \mathcal{O}}\left(N, \mathcal{O},\left\{m^{2}\right\}, \mu_{\mathrm{F}}^{2}, \mu_{\mathrm{R}}^{2}\right)\right. \\
& \left.\quad-\left.\frac{d \tilde{\hat{\sigma}}_{i j \rightarrow k l B}^{(\mathrm{res})}}{d \mathcal{O}}\left(N, \mathcal{O},\left\{m^{2}\right\}, \mu_{\mathrm{F}}^{2}, \mu_{\mathrm{R}}^{2}\right)\right|_{(\mathrm{NLO})}\right],
\end{aligned}
$$

where "res" can refer to either NLL or NNLL accuracy. Correspondingly, "matched" corresponds to NLO + N(N)LL predictions, i.e. $\mathrm{N}(\mathrm{N}) \mathrm{LL}$ resummed results matched to NLO, either only to NLO in QCD or to complete NLO QCD and EW corrections. Additionally, we include results at the accuracy referred to as "NLLwC"1, where the $N$ independent $\mathcal{O}\left(\alpha_{\mathrm{s}}\right)$ contributions in the expansion of the hard and soft functions, formally giving terms beyond NLL accuracy, are also included, see $[52,55]$ for a detailed description. The moments of the parton distribution functions $f_{i / h}\left(x, \mu_{\mathrm{F}}^{2}\right)$ are defined in the standard way

$f_{i / h}^{(N)}\left(\mu_{\mathrm{F}}^{2}\right) \equiv \int_{0}^{1} d x x^{N-1} f_{i / h}\left(x, \mu_{\mathrm{F}}^{2}\right)$,

and $d \hat{\sigma}_{i j \rightarrow k l B}^{(\text {res })} /\left.d Q^{2}\right|_{(\mathrm{NLO})}$ represents the perturbative expansion of the resummed cross section truncated at NLO. The inverse Mellin transform (4) is evaluated numerically using a contour $\mathrm{C}$ in the complex- $N$ space according to the "Minimal Prescription" method proposed in Ref. [65].

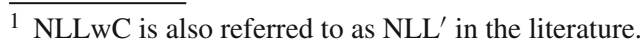


We include the electroweak effects additively while matching the resummed QCD calculation to the differential cross sections calculated at the complete NLO QCD and EW accuracy [66], from now indicated by NLO (QCD + EW). More specifically, this means that at the LO accuracy, apart from the $\mathcal{O}\left(\alpha_{\mathrm{s}}^{2} \alpha\right)$ contributions, also the $\mathcal{O}\left(\alpha_{\mathrm{s}} \alpha^{2}\right)$ and $\mathcal{O}\left(\alpha^{3}\right)$ terms are included. The complete NLO(QCD + EW) result, besides the $\mathcal{O}\left(\alpha_{\mathrm{s}}^{3} \alpha\right)$ correction, contains also the $\mathcal{O}\left(\alpha_{\mathrm{s}}^{2} \alpha^{2}\right), \mathcal{O}\left(\alpha_{\mathrm{s}} \alpha^{3}\right)$ and $\mathcal{O}\left(\alpha^{4}\right)$ corrections as well as the above-mentioned LO terms. Thus the EW effects are included in our final predictions up to NLO in the fine structure constant $\alpha$. It has been shown [62] that the differences between additive and multiplicative matching is small with the exception of extreme tails of the differential distributions, where the electroweak Sudakov and soft gluon logarithms both play a role. As we do not show predictions within the range where these effects become significant, the method of matching carries little relevance in the following.

Since we disentangle the pdfs from the partonic cross section by means of the Mellin transformation, there is no access to the individual $x_{1}$ and $x_{2}$ fractions of momenta of incoming partons. Therefore the formalism is restricted to observables invariant under boosts from the hadronic centerof-mass frame to the partonic center-of-mass frame, or correspondingly observables in the partonic frame. For example this means the resummation cannot be performed for a single particle rapidity distribution, but instead it can be performed for two particle rapidity difference distributions.

It is important to note that the threshold variable is not adapted for each individual observable. Therefore the threshold logarithms themselves do not depend on any observable other than the invariant mass of the three final state particles. The dependence on the other kinematic observables enters in the LO cross section, virtual corrections and the soft function. For the case of the $2 \rightarrow 2$ process of heavy quark production, there exists an alternative formulation of threshold resummation using the 1 particle inclusive (1PI) kinematics [67] instead of the pair-invariant mass (PIM) kinematics, an extension of which to triple particle production we are using here. The 1PI kinematic is naturally more suitable to describe certain observables, such as transverse momentum of one of the produced heavy quarks. However, while the analytical expressions for resummed distributions are known, the numerical calculations of the all-order resummed cross sections have not yet been achieved in direct QCD, even for $2 \rightarrow 2$ processes. In the next section we check how well our expanded resummed results approximate the NLO distributions. The checks make us confident that the three-particle invariant-mass resummation formalism is sufficient for these observables.

\section{Numerical results}

In this section we discuss in detail numerical results obtained for the total cross sections and differential distributions for the process $p p \rightarrow t \bar{t} B(B=H, Z, W)$ at $\sqrt{S}=13$ $\mathrm{TeV}$. Unless otherwise stated, all resummed results presented here include EW corrections implemented additively, i.e. through matching of the $\mathrm{NLO}(\mathrm{QCD}+\mathrm{EW})$ to the $\mathrm{N}(\mathrm{N}) \mathrm{LL}$ result, as explained above, and are called $\mathrm{NLO}(\mathrm{QCD}+\mathrm{EW})+\mathrm{NLL}, \mathrm{NLO}(\mathrm{QCD}+\mathrm{EW})+\mathrm{NLLwC}$ or $\mathrm{NLO}(\mathrm{QCD}+\mathrm{EW})+\mathrm{NNLL}$, correspondingly.

In order to estimate the sensitivity of the theoretical predictions to the choice of renormalization and factorization scales, and cross sections and distributions are calculated for different central scale choices $\mu_{0}$ :

$$
\begin{aligned}
& \mu_{0}=Q=\sqrt{\left(\sum_{i=t, \bar{t}, B} p_{i}\right)^{2}}, \\
& \mu_{0}=\frac{Q}{2}, \\
& \mu_{0}=\frac{M}{2}=\frac{\sum_{i=t, \bar{t}, B} m_{i}}{2}, \\
& \mu_{0}=H_{T}=\sum_{i=t, \bar{t}, B} m_{T}(i)=\sum_{i=t, \bar{t}, B} \sqrt{m_{i}^{2}+p_{T}^{2}(i)}, \\
& \mu_{0}=\frac{H_{T}}{2} .
\end{aligned}
$$

The invariant mass $Q$ can be seen as a natural scale for the kinematics of the invariant mass threshold resummation, whereas $H_{T}$-related scales are a popular dynamical scale choice in calculations of differential quantities, see e.g. [68]. Predictions for the total NLO cross sections are often provided in the literature for the fixed scale choice $\mu_{0}=M / 2$ [16-21,69]. The results for the total cross sections and invariant mass distributions at NLO(QCD) + NNLL for the scale choices $\mu_{0}=Q, \mu_{0}=M / 2$ and the "in between" scale choice $\mu_{0}=Q / 2$ were reported in [52,53] and in [55] for the $t \bar{t} H$ and $t \bar{t} V$ production, respectively. We also presented first results for the $p_{T}$ distribution of the $Z$ bosons in the process $p p \rightarrow t \bar{t} Z$, including results for $\mu_{0}=H_{T} / 2$, in [57].

The scale uncertainty for any prediction is estimated with the so-called 7-point method. All values are calculated for seven different pairs of $\mu_{\mathrm{F}}$ and $\mu_{\mathrm{R}}$ around the central scale $\mu_{0}$. The maximal and the minimal values of the seven combinations

$$
\begin{aligned}
& \left(\frac{\mu_{\mathrm{F}}}{\mu_{0}}, \frac{\mu_{\mathrm{R}}}{\mu_{0}}\right) \\
& \quad=(0.5,0.5),(0.5,1),(1,0.5),(1,1),(1,2),(2,1),(2,2)
\end{aligned}
$$


give then the uncertainty around the central value at $\left(\frac{\mu_{\mathrm{F}}}{\mu_{0}}, \frac{\mu_{\mathrm{R}}}{\mu_{0}}\right)$ $=(1,1)$.

In these numerical studies, we use the same input parameters as in the Higgs cross section working group (HXSWG) Yellow Report 4 [68] and our previous publication [55]: $m_{H}=125 \mathrm{GeV}, m_{t}=172.5 \mathrm{GeV}, m_{W}=$ $80.385 \mathrm{GeV}, m_{Z}=91.188 \mathrm{GeV}, G_{F}=1.1663787 \cdot 10^{-5}$ $\mathrm{GeV}^{-2}$. The CKM matrix in the calculations of the $t \bar{t} W$ cross sections is taken diagonal, in accordance with the Yellow Report setup. Concerning parton distribution functions, we use the PDF4LHC15_nlo_30 set [70-75] for NLO(QCD) cross sections and the NNLL results expanded up to $\mathcal{O}\left(\alpha_{\mathrm{s}}^{3}\right)$. The $\mathrm{NLO}(\mathrm{QCD}+\mathrm{EW})$ cross sections, as well as resummed results matched to them, are calculated with the LUXqed17_plus_PDF4LHC15_nnlo_100 set [70-77]. The pdf error is only calculated for the NLO cross section since the resummation is not expected to influence the value of the pdf error in any significant way.

The squared LO (QCD) amplitudes for $t \bar{t} W^{+} / W^{-} / Z$ in the multiplet basis were calculated with FORM [78] and the colour package [79] and then cross checked with MadGraph5_amc@NLO [31] and PowHel [33,35]. NLO cross sections were obtained with MadGraph5_aMC@NLO $[31,66]$. The QCD one loop virtual corrections needed for the hard colour matrix $\mathbf{H}^{(1)}$ were numerically extracted from PowHel and MadGraph5_aMC@NLO.

All numerical results for resummed quantities were calculated and cross-checked with two independent in-house Monte Carlo codes. We have checked that we reproduce the values for the $\mathrm{NLO}(\mathrm{QCD}+\mathrm{EW})$ total cross sections quoted in [66] and in [62] in the corresponding setups.

\subsection{Total cross sections}

We begin with the discussion of our results for the total cross sections for all three processes of associated top production with a heavy boson in $p p$ collisions at $\sqrt{S}=13 \mathrm{TeV}$. They are listed in Table 1 and graphically presented in Fig. 1. In order to keep the notation brief, we exceptionally refer to the NLO(QCD + EW) cross sections in Table 1 and in Fig. 1 as "NLO". For the predictions involving pure QCD corrections we refer the reader to $[52,56]$ and [55]. There we have also studied the quality of the approximation of the NLO total cross section provided by the $\mathcal{O}\left(\alpha_{\mathrm{s}}\right)$ expansion of the resummed expression and concluded that a big part of higher order corrections is indeed included in the resummed cross sections. Due to a similar impact of resummation on the $t \bar{t} W^{+}$and $t \bar{t} W^{-}$cross sections, for brevity we only show here results for their sum $(t \bar{t} W)$.

Our NLO $(\mathrm{QCD}+\mathrm{EW})+\mathrm{NNLL}$ results demonstrate remarkable stability w.r.t. different scale choices, delivering a compelling improvement of the theoretical predictions, specifically for processes involving a gluon channel. We see that the spread among the central values of the predictions, clearly visible at NLO(QCD + EW), is almost entirely eliminated for the $t \bar{t} Z$ and $t \bar{t} H$ production processes. Moreover, for all scale choices, the scale uncertainty is reduced as the accuracy of the calculation improves. The degree of the improvement varies depending on the central scale, as well as the process, reaching up to a factor of almost two. The effects are qualitatively similar, though less pronounced, for the $t \bar{t} W$ process.

Comparing the NLO $(\mathrm{QCD}+\mathrm{EW})+\mathrm{NNLL}$ cross sections obtained using the LUXqed17_ plus_PDF4 LHC15_nnlo_ 100 pdf set with the NLO (QCD) + NNLL predictions obtained using the PDF4LHC15_nnlo_30 set $[55,56]$ we observe that for the $t \bar{t} H$ and the $t \bar{t} W$ production the EW effects lead to an increase (albeit very small, ca. $1 \%$, in the $t \bar{t} H$ case) in the total cross sections, whereas the results for the $t \bar{t} Z$ production get only minimally affected and the differences are within the size of our statistical Monte Carlo uncertainty. This behaviour is inherited from the NLO(QCD $+\mathrm{EW})$ and $\mathrm{NLO}(\mathrm{QCD})$ results, where in most cases the EW effects (obtained using a corresponding pdf set) lead to positive corrections, in agreement with [66]. Since the EW corrections are introduced additively into the matched formula, c.f. Eq. (4), the effects of resummation are very similar to the pure QCD case. Correspondingly, the NNLL K-factors, i.e. ratios of the $\mathrm{NLO}(\mathrm{QCD}+\mathrm{EW})+\mathrm{NNLL}$ total cross sections to the $\mathrm{NLO}(\mathrm{QCD}+\mathrm{EW})$ total cross sections listed in Table 1 and the ones obtained for the pure QCD cross sections quoted in [52], [55] and [56] are very similar as the EW corrections impact them only minimally.

Given the observed improvement in stability of the predictions w.r.t. scale variation at $\mathrm{NLO}(\mathrm{QCD}+\mathrm{EW})+\mathrm{NNLL}$, we combine the predictions for our five scale choices, according to the envelope method proposed in [69]. The corresponding results are

$\begin{aligned} \sigma_{t \bar{t} H}^{\mathrm{NLO}+\mathrm{NNLL}} & =504_{-7.1 \%-2.4 \%}^{+7.6 \%+2.4 \%} \mathrm{fb}, \\ \sigma_{t \bar{t} Z}^{\mathrm{NLO}+\mathrm{NNLL}} & =859_{-9.5 \%-2.3 \%}^{+8.6 \%+2.3 \%} \mathrm{fb}, \\ \sigma_{t \bar{t} W}^{\mathrm{NLO}+\mathrm{NNLL}} & =592_{-16.2 \%-2.1 \%}^{+26.1 \%+2.1 \%} \mathrm{fb}\end{aligned}$

at $\sqrt{S}=13 \mathrm{TeV}$. The first error is the scale uncertainty while the second one is the PDF uncertainty of the NLO(QCD + EW) prediction.

As mentioned in the Introduction, predictions for selected differential $t \bar{t} B$ distributions at the NLO $(\mathrm{QCD}+\mathrm{EW})+$ NNLL accuracy were also calculated in the SCET approach [62]. In principle, a comparison of results obtained in the two different approaches not only can be used as an independent check of the two calculations, but also deliver information on the size of the effects which are formally below the considered level of precision. It has to be noted though 
Table 1 Predictions for the total $p p \rightarrow t \bar{t} H / Z / W$ cross section at $\sqrt{S}=13 \mathrm{TeV}$ and different $\mu_{0}$. "NLO" stands here for $\mathrm{NLO}(\mathrm{QCD}+\mathrm{EW})$. The listed error is the scale uncertainty calculated with the 7-point method

\begin{tabular}{lllllll}
\hline Process & $\mu_{0}$ & NLO[fb] & NLO+NLL[fb] & NLO+NLLwC[fb] & NLO + NNLL[fb] & $K_{\text {NNLL }}$ \\
\hline$t \bar{t} H$ & $Q$ & $425_{-11.6 \%}^{+12.1 \%}$ & $445_{-9.2 \%}^{+10.0 \%}$ & $489_{-8.5 \%}^{+8.4 \%}$ & $505_{-7.0 \%}^{+7.5 \%}$ & 1.19 \\
& $H_{T}$ & $434_{-11.4 \%}^{+11.6 \%}$ & $451_{-8.9 \%}^{+9.5 \%}$ & $491_{-8.2 \%}^{+7.9 \%}$ & $502_{-6.7 \%}^{+7.3 \%}$ & 1.16 \\
& $Q / 2$ & $476_{-10.8 \%}^{+9.9 \%}$ & $484_{-8.2 \%}^{+8.7 \%}$ & $503_{-7.3 \%}^{+6.2 \%}$ & $505_{-6.4 \%}^{+5.7 \%}$ & 1.06 \\
& $H_{T} / 2$ & $484_{-10.4 \%}^{+8.9 \%}$ & $490_{-8 \%}^{+8.4 \%}$ & $503_{-6.8 \%}^{+5.5 \%}$ & $502_{-6.1 \%}^{+5.4 \%}$ & 1.04 \\
& $M / 2$ & $506_{-9.3 \%}^{+6 \%}$ & $510_{-7.8 \%}^{+8.2 \%}$ & $512_{-6.2 \%}^{+5.9 \%}$ & $510_{-6.1 \%}^{+5.6 \%}$ & 1.01 \\
& $Q \bar{t} Z$ & $661_{-12.5 \%}^{+13.8 \%}$ & $698_{-10.1 \%}^{+11.5 \%}$ & $795_{-9.7 \%}^{+10.6 \%}$ & $847_{-8.2 \%}^{+8.1 \%}$ & 1.28 \\
& $H_{T}$ & $694_{-12.6 \%}^{+13.6 \%}$ & $723_{-9.8 \%}^{+11.0 \%}$ & $805_{-9.5 \%}^{+10.0 \%}$ & $848_{-8.0 \%}^{+7.9 \%}$ & 1.22 \\
& $Q / 2$ & $752_{-12.1 \%}^{+12.5 \%}$ & $770_{-9.4 \%}^{+10.6 \%}$ & $824_{-8.8 \%}^{+8.8 \%}$ & $854_{-7.8 \%}^{+7.1 \%}$ & 1.14 \\
& $H_{T} / 2$ & $788_{-11.9 \%}^{+11.7 \%}$ & $798_{-9.5 \%}^{+10.7 \%}$ & $834_{-8.4 \%}^{+8.1 \%}$ & $855_{-7.7 \%}^{+6.6 \%}$ & 1.09 \\
& $M / 2$ & $841_{-11.1 \%}^{+9.4 \%}$ & $848_{-9.7 \%}^{+11.2 \%}$ & $858_{-7.9 \%}^{+7.1 \%}$ & $874_{-7.8 \%}^{+6.7 \%}$ & 1.04 \\
& $Q$ & $512_{-11.1 \%}^{+12.5 \%}$ & $516_{-10.6 \%}^{+12.1 \%}$ & $533_{-8.9 \%}^{+9.9 \%}$ & $541_{-8.4 \%}^{+8.9 \%}$ & 1.06 \\
& $H_{T}$ & $539_{-11.3 \%}^{+13.0 \%}$ & $542_{-10.9 \%}^{+12.6 \%}$ & $556_{-9.0 \%}^{+10.5 \%}$ & $562_{-8.5 \%}^{+9.6 \%}$ & 1.04 \\
& $Q / 2$ & $577_{-11.1 \%}^{+12.5 \%}$ & $579_{-10.8 \%}^{+12.3 \%}$ & $586_{-9.0 \%}^{+10.7 \%}$ & $590_{-8.5 \%}^{+10.0 \%}$ & 1.02 \\
& $H_{T} / 2$ & $609_{-11.5 \%}^{+13.0 \%}$ & $610_{-11.2 \%}^{+13 \%}$ & $614_{-9.5 \%}^{+11.8 \%}$ & $616_{-8.8 \%}^{+1.2 \%}$ & 1.01 \\
$M / 2$ & $656_{-11.7 \%}^{+13.2 \%}$ & $658_{-11.6 \%}^{+13.6 \%}$ & $657_{-10.3 \%}^{+13.4 \%}$ & $659_{-9.8 \%}^{+13.3 \%}$ & 1.00 \\
\hline
\end{tabular}

Fig. 1 Graphical illustration of the predictions in Table 1.

"NLO" stands here for $\mathrm{NLO}(\mathrm{QCD}+\mathrm{EW})$
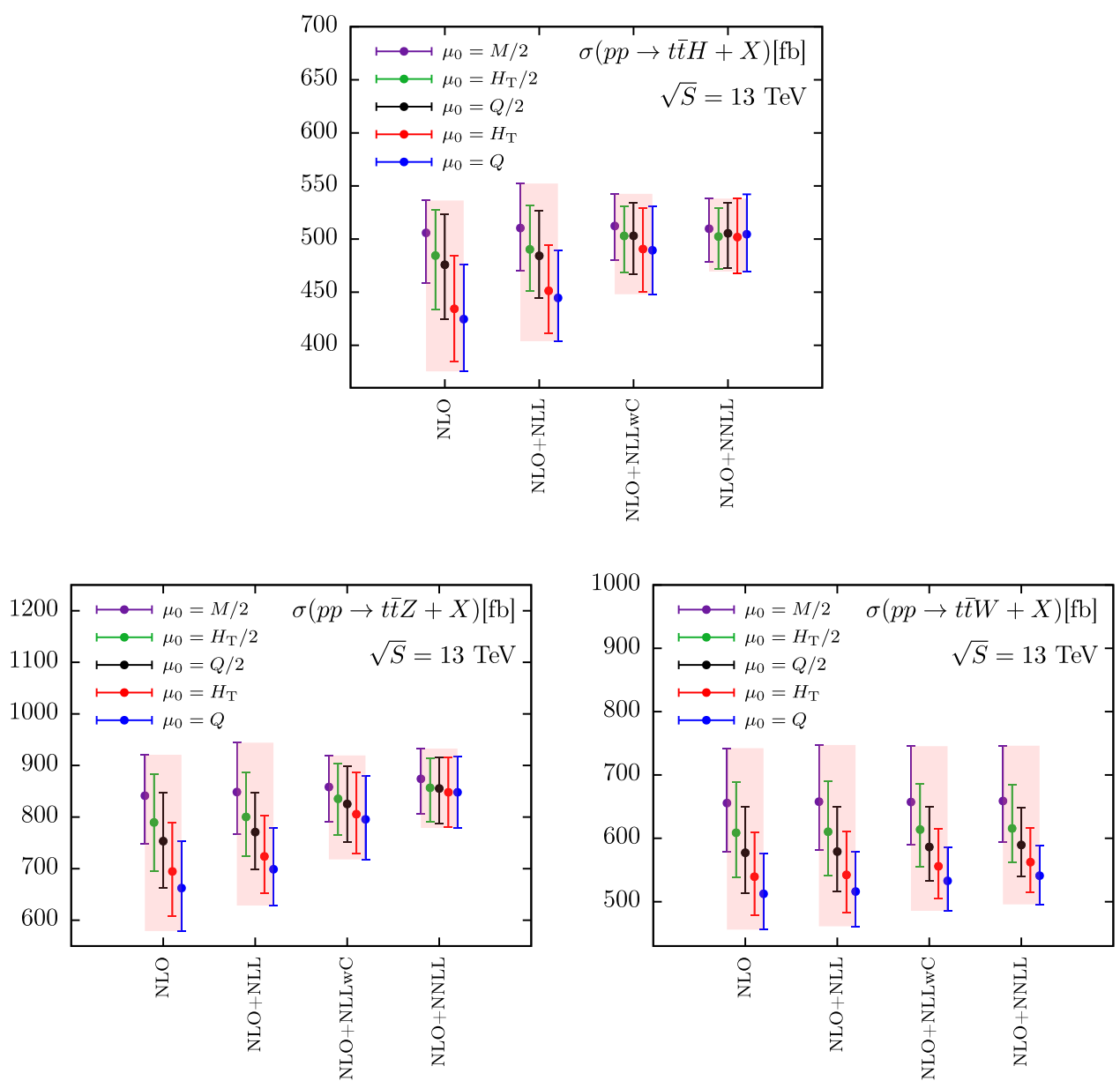
that the two approaches involve two different sets of scales: our direct QCD calculations depend only on the factorization and renormalization scales, $\mu_{\mathrm{F}}$ and $\mu_{\mathrm{R}}$, while the SCET formalism involves the factorization, soft and hard scales, $\mu_{\mathrm{F}}$, $\mu_{S}$ and $\mu_{H}$. Unfortunately, the treatment of the scales in the resummed and expanded parts as well as the scale choices made in [62]: the $Q$-based $\left\{\mu_{\mathrm{F}}=Q / 2, \mu_{H}=Q, \mu_{S}=\right.$ $Q / \bar{N}\}$ and the $H_{T}$-based $\left\{\mu_{\mathrm{F}}=H_{T} / 2, \mu_{H}=H_{T} / 2, \mu_{S}=\right.$ $\left.H_{T} \bar{N}\right\}$ sets cannot be directly translated into corresponding choices of $\left\{\mu_{\mathrm{F}}, \mu_{\mathrm{R}}\right\}$. Consequently, no meaningful conclusions on the impact of subleading terms can be drawn by comparing only the central values. However, one can compare an overall behavior of the results as well as the values of the cross sections within their scale errors. We also need to point out that the error estimates are performed in different way. While we use the seven-point method, in Ref. [62] each of the three scales, $\mu_{\mathrm{F}}, \mu_{H}$ and $\mu_{S}$ is varied independently w.r.t. its central value by a factor of $2^{ \pm 1}$, while the two other scales are frozen. The total uncertainty is then obtained by adding in quadrature the deviations corresponding to these three scales. Finally, as the most conservative estimate of the theoretical uncertainty, in the present study the envelope method is applied to five central scale choices: $\mu_{0}=Q, H_{T}$, $Q / 2, H_{T} / 2, M / 2$, in contrast to the two ( $Q$-based and $H_{T^{-}}$ based) choices in Ref. [62]. In all cases, the estimates of the pdf uncertainties agree at per mille point precision and will be not discussed further.

Using the scale envelope scale uncertainty, at NLO(QCD+ $\mathrm{EW})+\mathrm{NNLL}$ we obtain $\sigma(t \bar{t} H)=504_{-7.1 \%}^{+7.6 \%} \mathrm{fb}$ vs $\sigma(t \bar{t} H)=$ $496_{-5.9 \%}^{+7.8 \%}$ fb in Ref. [62]. The results agree well within the error bars and the theoretical uncertainties are also close in value to each other. Although the results for $\sigma(t \bar{t} Z)$ also agree within errors, we find here a bigger difference, i.e. $\sigma(t \bar{t} Z)=$ $859_{-9.5 \%}^{+8.6 \%} \mathrm{fb}$ vs $\sigma(t \bar{t} Z)=811_{-9.6 \%}^{+11.0 \%}$ in Ref. [62]. If we adjust the value of the top quark mass to the same value as used in Ref. [62] $\left(m_{t}=173.34 \mathrm{GeV}\right)$, the envelope value of our result reduces to $846_{-9.5 \%}^{+8.3 \%} \mathrm{fb}$. Nevertheless, the percentage difference between our results and that of Ref. [62] is bigger for $\sigma(t \bar{t} Z)$ than for $\sigma(t \bar{t} H)$. This can be traced back to the overall higher $K_{\mathrm{NNLL}}$ values for the $t \bar{t} Z$ process, as well as a bigger spread of $K_{\mathrm{NNLL}}$ values in our approach. The latter leads to a much smaller spread of our NLO $(\mathrm{QCD}+\mathrm{EW})+$ NNLL predictions compared to Ref. [62], corresponding to a difference in stability of the NLO(QCD + EW $)+$ NNLL results between $1 \%$ level for our calculations and $3 \%$ for [62].

For the $t \bar{t} W$ production we obtain $\sigma(t \bar{t} W)=592_{-16.2 \%}^{+26.1 \%} \mathrm{fb}$ vs $\sigma(t \bar{t} W)=582_{-8.2 \%}^{+13.4 \%} \mathrm{fb}$ in Ref. [62], where we added the predictions for $t \bar{t} W^{+}$and $t \bar{t} W^{-}$. Here the central values are pretty close but we estimate the theoretical uncertainties to be significantly larger. This difference can be unambiguously explained by the wider central scale range used in our envelope. When the scale decreases from $Q$ to $M / 2$, the
$\mathrm{NLO}(\mathrm{QCD}+\mathrm{EW})$ cross section grows by about $28 \%$. For a relatively smaller central scales span between $H_{T} / 2$ and $Q / 2$ used in [62], the difference in the $\mathrm{NLO}(\mathrm{QCD}+\mathrm{EW})$ cross section is only about $5.5 \%$. In both approaches, the impact of resummation is moderate, ranging from about $0.5 \%$ at $\mu_{0}=M / 2$ to about $5 \%$ at the highest scale $Q$ in our case and from 1 to $2 \%$ in [62]. Consequently, the method of estimating the uncertainty of the $\mathrm{NLO}(\mathrm{QCD}+\mathrm{EW})$ result leaves its imprint on the uncertainty of the NLO $(\mathrm{QCD}+\mathrm{EW})+\mathrm{NNLL}$ predictions. However, while we find that the resummation brings the central values of the predictions obtained with various scale choices closer together, Ref. [62] reports that $\mathrm{NLO}(\mathrm{QCD}+\mathrm{EW})+\mathrm{NNLL}$ predictions for $Q$-based and $H_{T}$ based scale choices are more spread apart than NLO(QCD + EW).

The main conclusion from the comparison of $\mathrm{NLO}(\mathrm{QCD}$ $+\mathrm{EW})+$ NNLL total cross sections in Ref. [62] and the present paper is that the results agree within the uncertainties. In most cases the estimated theoretical uncertainties are also similar, with one exception of $t \bar{t} W$ where there is a significant difference of scale uncertainties due to different ranges of central scales taken into account in the two approaches.

\subsection{Differential distributions}

In the following we present differential distributions for the processes $p p \rightarrow t \bar{t} B(B=H, Z, W)$. More specifically, these are distributions in the invariant mass $Q$ of the $t \bar{t} B$ system, the invariant mass $m_{t \bar{t}}$ of the $t \bar{t}$ pair, transverse momentum of the top quark $p_{T}(t)$, transverse momentum of the boson $p_{T}(B)$, the difference in rapidities between the top quark and the antitop quark $y(t)-y(\bar{t})$, the difference in rapidities between the top quark and the boson $y(t)-y(B)$, the difference in the azimuthal angle between the top quark and the antitop quark $\phi(t)-\phi(\bar{t})$ and the difference in the azimuthal angle between the top quark and the boson $\phi(t)-\phi(B)$.

In [52] and [55], we showed that the resummed results for the total cross sections, expanded to the same order in $\alpha_{\mathrm{s}}$ as that of NLO, approximates well the full NLO(QCD) cross section in the $q \bar{q}$ and $g g$ channels, especially for the $t \bar{t} H$ and $t \bar{t} Z$ production. The $q g$ channel appears for the first time at NLO and no resummation is performed for this channel: it only enters the resummation-improved predictions via matching. Before commenting on individual differential distributions we first study if the statement regarding the quality of the approximation carries on to the differential level. Since the effects of higher order logarithmic corrections treated by resummation are very similar for the $t \bar{t} H$ and $t \bar{t} Z$ production and are very similar for these two processes, we present a corresponding study only for the $t \bar{t} Z$ process. We choose to analyze the quality of the approximation of the NLO(QCD) distributions by the expansion of the NNLL result at the scale 
Fig. 2 Comparison between the expansion of the NNLL expression up to NLO accuracy in $\alpha_{\mathrm{s}}$, the full $\mathrm{NLO}(\mathrm{QCD})$ result and the NLO(QCD) result without the $q g$ channels for the $p p \rightarrow t \bar{t} Z$ differential distributions in $Q$ and $m_{t \bar{t}}$

Fig. 3 The same as in Fig. 2 but for the $p p \rightarrow t \bar{t} Z$ differential distributions in $p_{T}(t)$ and $p_{T}(Z)$
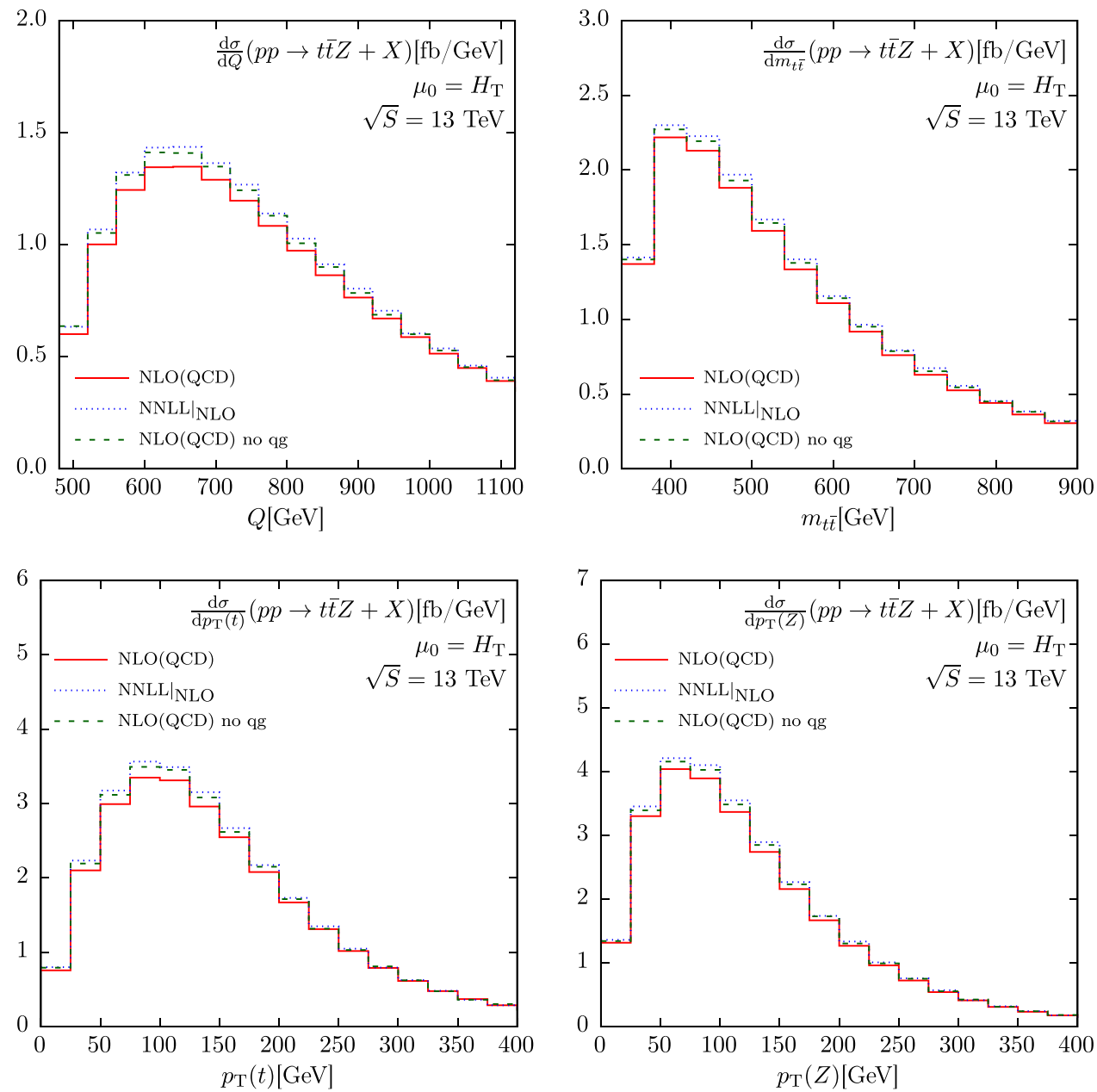

$\mu_{0}=H_{T}$ where the resummation effects are very relevant. The results for all differential distributions mentioned above are shown in Figs. 2, 3, 4 and 5 . Note that in this comparison the $\mathrm{NLO}(\mathrm{QCD})$ cross sections are used, as the judgement of the quality of the approximation concerns only the QCD corrections. We observe that the expanded NNLL differential distributions offer very good approximations of the $\mathrm{NLO}(\mathrm{QCD})$ results for the distributions in $Q, m_{t \bar{t}}, p_{T}(t)$, $p_{T}(Z), y(t)-y(\bar{t})$ and $y(t)-y(Z)$. For the distributions in $\phi(t)-\phi(\bar{t})$ and $\phi(t)-\phi(Z)$, the quality of the approximation is excellent for small angle differences. It worsens slightly with increasing angle but without exceeding $5 \%$ difference in the largest angular difference bins. With this small exception, the demonstrated quality of the approximation let us conclude that the NNLL differential distributions for the $t \bar{t} Z$ production considered here take into account a big part of the higher order corrections to the $g g$ and $q \bar{q}$ channels. Next, we perform analogous studies for the process $p p \rightarrow t \bar{t} W$, see Figs. 6, 7, 8 and 9. Also in this case we observe that the expanded NNLL result provides a very good approximation of the NLO distributions when the $q g$ channel contributions are subtracted.
The NLO(QCD + EW $)$ + NNLL distributions are presented in Figs. 10, 11, 12 and 13 for the $t \bar{t} H$ production, Figs. 14, 15, 16 and 17 for the $t \bar{t} Z$ production and Figs. 18, 19,20 and 21 for the $t \bar{t} W$ production. We choose to show results for three representative scale choices: $\mu_{0}=M / 2$, $\mu_{0}=Q / 2$ and $\mu_{0}=H_{T}$. With the total cross section results for $\mu_{0}=H_{T}$ and $\mu_{0}=Q$ being very close, we expect that such a choice of central scales covers the span of theoretical uncertainty for the predictions well. Results for NNLL differential distribution in $Q$, matched to NLO (QCD), were previously discussed by us in $[52,56]$ for the $t \bar{t} H$ production and in [55] for the $t \bar{t} Z$ and $t \bar{t} W$ production. Here the NNLL results are matched to the NLO(QCD + EW) predictions and although there is not much difference in the behaviour of the $d \sigma / d Q$ cross section due to the presence of the EW corrections, we include the $d \sigma / d Q$ cross section at $\mathrm{NLO}(\mathrm{QCD}+\mathrm{EW})+\mathrm{NNLL}$ in our presentation for completeness. The top panels of Figs. 10, 11, 12, 13, 14, 15,16 and 17 show an excellent agreement for the $t \bar{t} H$ and $t \bar{t} Z \mathrm{NLO}(\mathrm{QCD}+\mathrm{EW})+\mathrm{NNLL}$ predictions obtained for the three scale choices. As the impact of higher order logarithmic corrections is weaker for the $t \bar{t} W$ cross sections, 
Fig. 4 The same as in Fig. 2 but for the $p p \rightarrow t \bar{t} Z$ differential distributions in $\phi(t)-\phi(\bar{t})$ and $\phi(t)-\phi(Z)$

Fig. 5 The same as in Fig. 2 but for the $p p \rightarrow t \bar{t} Z$ differential distributions in $y(t)-y(\bar{t})$ and $y(t)-y(Z)$
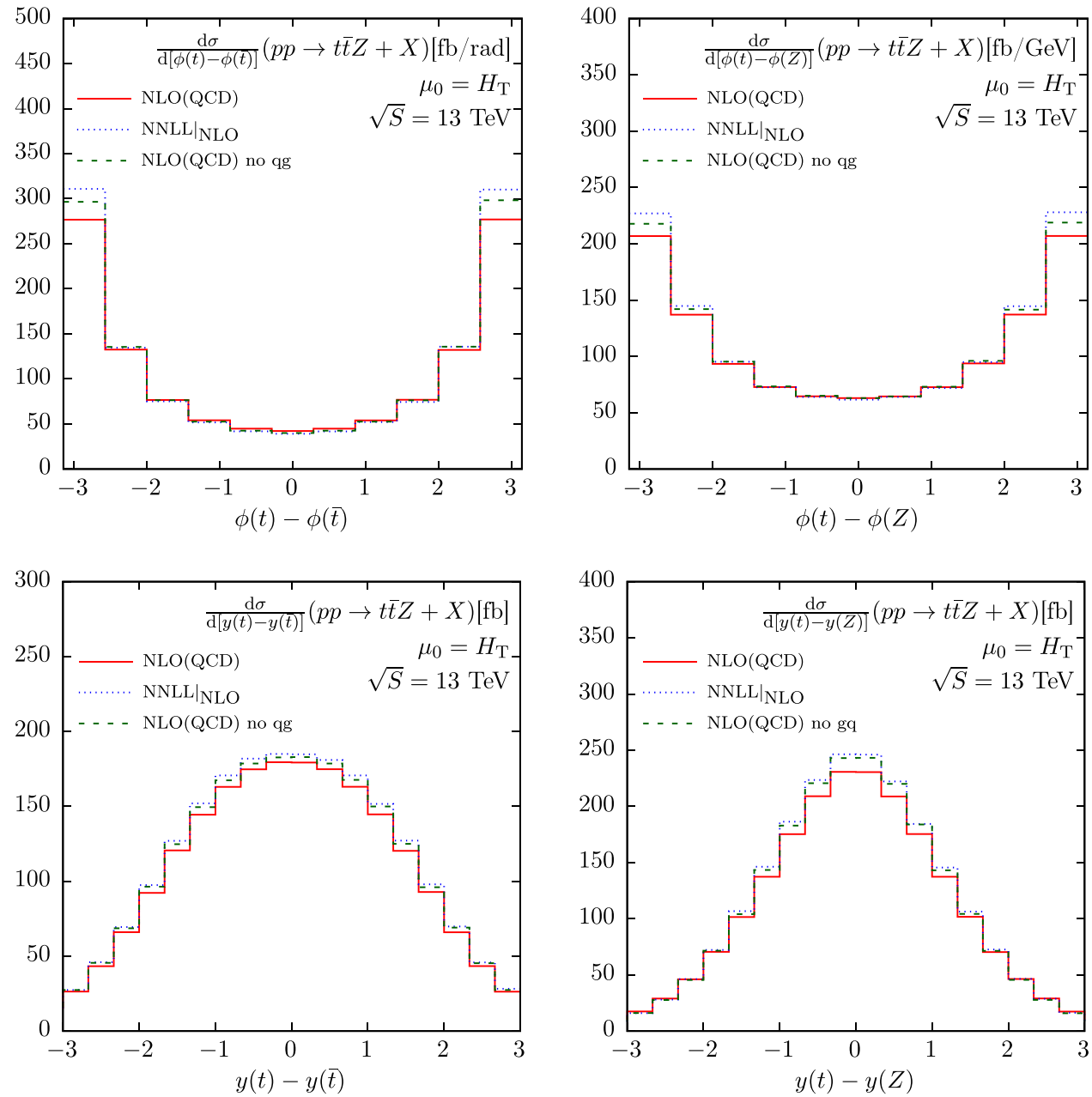

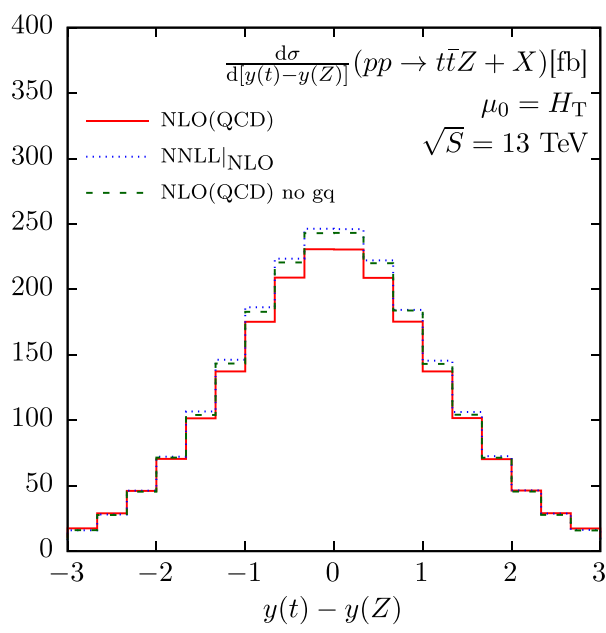

the spread of predictions for $t \bar{t} W$ distributions does not get substantially decreased by adding NNLL resummation. The relatively small effect of the NNLL corrections on the $t \bar{t} W$ distributions is in line with the behaviour of the total cross sections, see Fig. 1 and Ref. [55]. In contrast to the $t \bar{t} H$ and $t \bar{t} Z$ processes, the $t t W$ production at LO involves only the $q \bar{q}^{\prime}$ channel. Correspondingly the NNLL resummation at leading power takes into account only the soft gluon emission from incoming quark lines which by means of colour factors is much weaker than the emission from gluon lines. The NNLL contributions are then simply too modest to outweigh the scale dependence of the NLO $(\mathrm{QCD}+\mathrm{EW})$ result to the same extent as they do for the $g g$ channel dominated processes, leading to a bigger spread in the central values of NLO(QCD + EW) NNLL predictions. This also explains why the reduction of the scale uncertainties for various central scale choices due to resummation is weaker for the $t t W$ production, compared to the $t \bar{t} H$ and $t \bar{t} Z$ processes.

The lower three panels in the figures show ratios of the $\mathrm{NLO}(\mathrm{QCD}+\mathrm{EW})+\mathrm{NNLL}$ distributions to the NLO$(\mathrm{QCD}$ + EW) distributions, i.e. the $K_{\mathrm{NNLL}}$ factor, calculated for different values of $\mu_{0}$. The dark shaded areas indicate the scale errors of the NLO $(\mathrm{QCD}+\mathrm{EW})+\mathrm{NNLL}$ predictions, while light-shaded areas correspond to the scale errors of the $\mathrm{NLO}(\mathrm{QCD}+\mathrm{EW})$ results. We observe that the ratios can differ substantially depending on the final state, observable or the central scale. Generally, the NNLL resummation has the biggest impact on the predictions obtained for $\mu_{0}=H_{T}$ among the three scale choice we study. In the case of the distributions in $Q, m_{t \bar{t}}, p_{T}(t), p_{T}(B), \phi(t)-\phi(\bar{t}), \phi(t)-\phi(B)$ the ratios show that resummation can contribute as much as ca. $20 \%(30 \%)$ correction to the $t \bar{t} H(t \bar{t} Z)$ distribution at this scale choice. As observed in [52] and in [55], the size of the NNLL corrections to the invariant distribution in $Q$ mildly increases with $Q$. The same can be seen for the distribution in $m_{t \bar{t}}$, c.f. Figs. 10 and 14. The $p_{T}(t)$ distributions, on the other hand, receive the biggest NNLL corrections towards smaller values of $p_{T}$, whereas the corrections to the the $p_{T}(H), p_{T}(Z)$ distributions get most pronounced for moderate values of $p_{T}$, see Figs. 11 and 15. From Figs. 12 and 16 it can be observed that the distributions in the difference between the azimuthal angles of the top and the antitop quark are impacted the most for collinear configurations for almost all scale choices. The difference between azimuthal angles 
Fig. 6 Comparison between the expansion of the NNLL expression up to NLO accuracy in $\alpha_{\mathrm{s}}$, the full $\mathrm{NLO}(\mathrm{QCD})$ result and the $\mathrm{NLO}(\mathrm{QCD})$ result without the $q g$ channels for the $p p \rightarrow t \bar{t} W$ differential distributions in $Q$ and $m_{t \bar{t}}$

Fig. 7 The same as in Fig. 6 but for the $p p \rightarrow t \bar{t} W$ differential distributions in $p_{T}(t)$ and $p_{T}(W)$
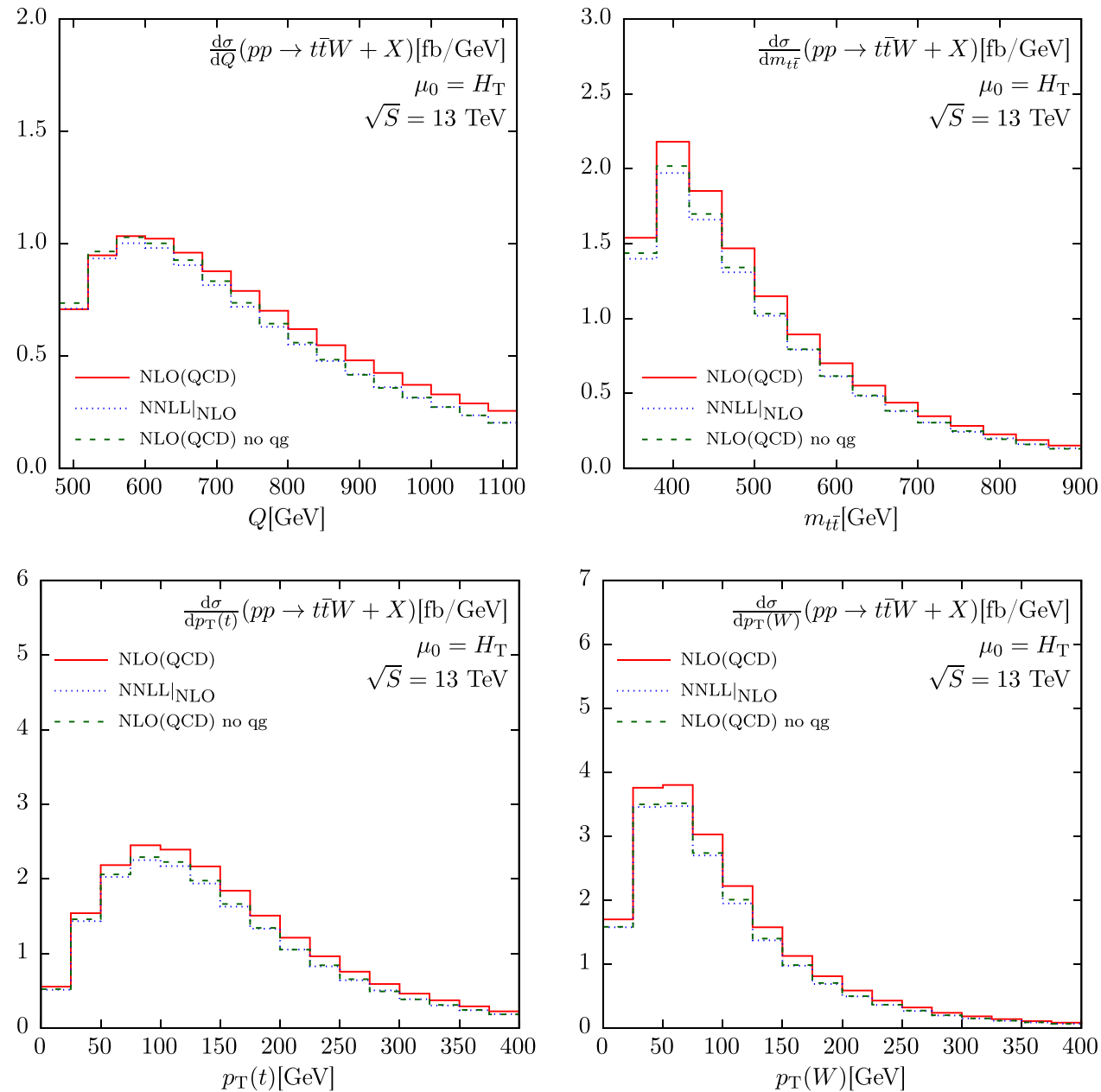

of the top and the $H$ or $Z$ boson show an opposite behaviour, i.e. they are enhanced for the back-to-back configurations in the transverse plane. The distributions in differences between rapidities, in particular $y(t)-y(\bar{t})$, can receive corrections of up to ca. $40 \%$, especially at high values of rapidity differences. These distributions also receive smaller NNLL corrections at lower rapidity differences, with the corrections generally growing as the difference in rapidity grow. For the reasons described above, the $t \bar{t} W$ distributions, on the other hand, get modified by a few percent, at most reaching up to $10 \%$, corrections.

The NNLL effects in the differential distributions are similar for the present paper and Ref. [62]. The comparison may be performed for the $Q, m_{t \bar{t}}, p T(V)$ and $p_{T}(t)$ distributions. Within the ranges of the variables considered here, the overall picture is similar in both frameworks. The NNLL corrections do not affect the shapes of the distributions strongly and exhibit only mild kinematical dependence. The typical effects of NNLL are positive and of order $10 \%$. The NNLL effects lead to a better theoretical precision of the prediction. The improvement is stronger for $t \bar{t} H$ and $t \bar{t} Z$, resulting in the error band at or below $10 \%$ level, and weaker in $t \bar{t} W$, where the uncertainty bands are close or above $15 \%$.

Regarding the comparison with other results in the literature, in particular Ref. [62], the same remarks as in the discussion of the total cross section apply: different scale set-ups and choices do not allow to draw conclusions on the impact of formally subleading terms but we can examine absolute values of differential cross sections. As an example, we compare the invariant mass $Q$ distributions obtained here with the corresponding ones from Ref. [62]. For this purpose we apply the envelope method to the bin situated at the peak of $Q$-distribution, at $Q=Q_{\max }$. The $K_{\mathrm{NNLL}}\left(Q_{\max }\right)$ factors obtained this way are compared with the values read off the plots in [62]. For the $t \bar{t} H$ production we obtain $K_{\mathrm{NNLL}}\left(Q_{\max }\right)=1.07_{-0.08}^{+0.08}$ which should be compared with $1.02_{-0.06}^{+0.08}$ from [62]. For the $t \bar{t} Z$ process we get $K_{\mathrm{NNLL}}\left(Q_{\max }\right)=1.13_{-0.10}^{+0.09}$ compared to $1.05_{-0.09}^{+0.11}$ from [62]. For the $t \bar{t} W$ process we get $K_{\mathrm{NNLL}}\left(Q_{\max }\right)=1.04_{-0.12}^{+0.13}$, whereas the authors of [62] obtain $0.99_{-0.09}^{+0.09}$ for $t \bar{t} W^{+} .{ }^{2} \mathrm{We}$

\footnotetext{
${ }^{2}$ Since the $K$-factors for $t \bar{t} W^{+}$and $t \bar{t} W^{-}$in Ref. [62] are very similar, we compare them with our $K_{\mathrm{NNLL}}$ for $t \bar{t} W$.
} 
Fig. 8 The same as in Fig. 6 but for the $p p \rightarrow t \bar{t} W$ differential distributions in $\phi(t)-\phi(\bar{t})$ and $\phi(t)-\phi(W)$

Fig. 9 The same as in Fig. 6 but for the $p p \rightarrow t \bar{t} W$ differential distributions in $y(t)-y(\bar{t})$ and $y(t)-y(W)$
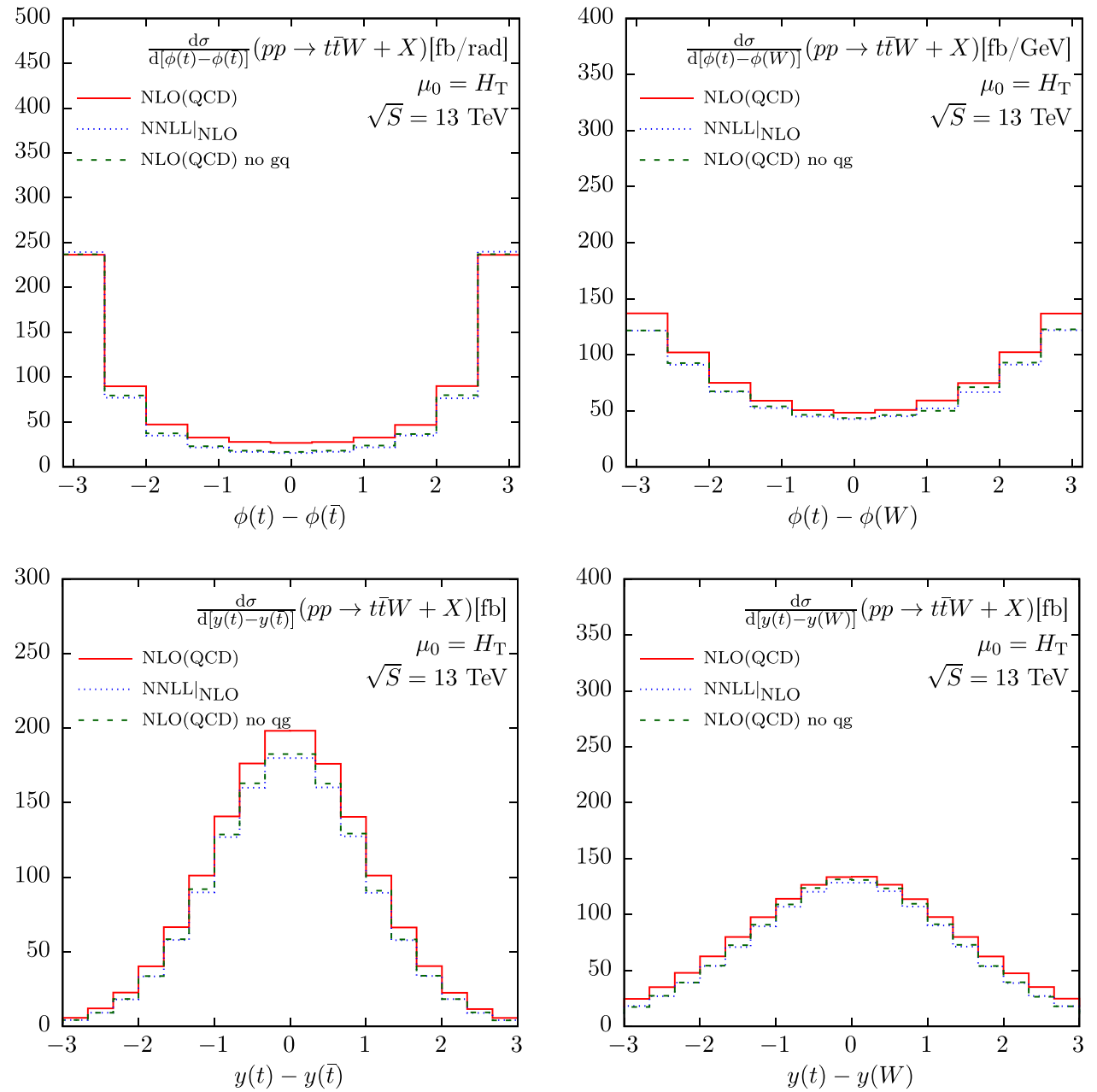

see a similar agreement to the one found in comparison for the inclusive cross section. The size of the resummation corrections lie within the scale uncertainty of one another. In addition the size of the scale uncertainties in the different approaches is comparable, with the exception of $t \bar{t} W$.

We finish the discussion by comparing our results for the differential distribution in $p_{T}(Z)$ with the very recent measurement of this distribution by the CMS collaboration [15]. In Fig. 22 the NLO(QCD + EW) predictions are compared with the NLO $(\mathrm{QCD}+\mathrm{EW})+\mathrm{NNLL}$ results for two different scales choices, $\mu_{0}=H_{T}$ and $\mu_{0}=Q / 2$. We see that the resummed NNLL corrections bring the theoretical predictions closer to data and lead to a significant reduction in the scale dependence error. The left plot in Fig. 23 shows the comparison of the NLO(QCD + EW $)+$ NNLL predictions for various scale choices, adjusted for the bin widths as used in the experimental measurement, while the right plot shows the same comparison for the shapes of the distributions. In accordance with observations made above, the NNLL calculations yield our predictions remarkable stable w.r.t. the scale variation.

\section{Summary}

In this paper we consider processes of associated top-antitop quark pair and a heavy boson $B=H, Z$ or $W^{ \pm}$production in $p p$ collisions. Theoretical predictions for total and differential cross sections at the LHC are obtained using the soft gluon resummation technique in Mellin space through the NNLL accuracy in QCD matched to existing NLO results in the QCD and the electroweak theory. The calculations are based on the framework developed in our earlier work [5057], and the main aim of the present study is to provide an accurate theoretical reference for a wide set of observables that are or may be measured in the $p p \rightarrow t \bar{t} H, p p \rightarrow t \bar{t} Z$ and $p p \rightarrow t \bar{t} W^{ \pm}$scattering at the LHC. The framework applied offers the currently best available theoretical precision, with the reduction of the theoretical uncertainties due to scale variation reaching up to a factor of about two with respect to the corresponding $\mathrm{NLO}(\mathrm{QCD}+\mathrm{EW})$ estimates.

The main focus of the present study are the differential cross sections. Hence we present the distributions of the $t \bar{t} B$ and $t \bar{t}$ invariant masses, $p_{T}$ of the boson and $p_{T}$ of the top quark. Moreover we obtain the distributions for the azimuthal 
Fig. 10 Predictions for $p p \rightarrow t \bar{t} H$ differential cross section in $Q$ and $m_{t \bar{t}}$. Lower panels show ratio of the $\mathrm{NLO}(\mathrm{QCD}+\mathrm{EW})+\mathrm{NNLL}$ and $\mathrm{NLO}(\mathrm{QCD}+\mathrm{EW})$ distributions for three central scale choices $\mu_{0}=M / 2, \mu_{0}=Q / 2$ and $\mu_{0}=H_{T}$. Only scale uncertainties are shown
Fig. 11 Predictions for $p p \rightarrow t \bar{t} H$ differential cross section in $p_{T}(t)$ and $p_{T}(H)$. Lower panels show ratio of the $\mathrm{NLO}(\mathrm{QCD}+\mathrm{EW})+\mathrm{NNLL}$ and $\mathrm{NLO}(\mathrm{QCD}+\mathrm{EW})$ distributions for three central scale choices $\mu_{0}=M / 2, \mu_{0}=Q / 2$ and $\mu_{0}=H_{T}$. Only scale uncertainties are shown
Fig. 12 Predictions for $p p \rightarrow t \bar{t} H$ differential cross section in $\phi(t)-\phi(\bar{t})$ and $\phi(t)-\phi(H)$. Lower panels show ratio of the NLO(QCD + $\mathrm{EW})+\mathrm{NNLL}$ and NLO(QCD + EW) distributions for three central scale choices $\mu_{0}=M / 2$, $\mu_{0}=Q / 2$ and $\mu_{0}=H_{T}$. Only scale uncertainties are shown
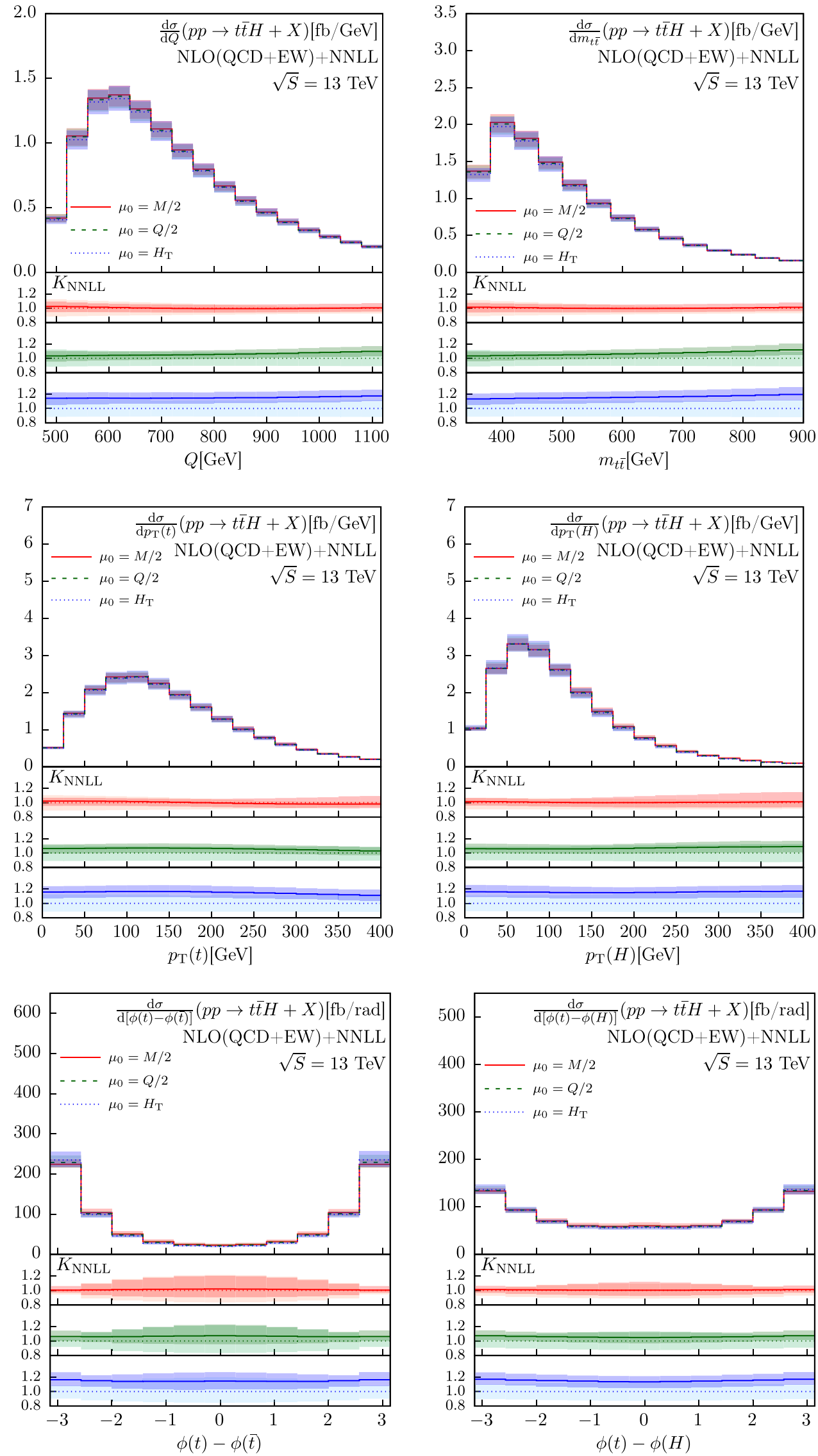
Fig. 13 Predictions for $p p \rightarrow t \bar{t} H$ differential cross section in $y(t)-y(\bar{t})$ and $y(t)-y(H)$. Lower panels show ratio of the $\mathrm{NLO}(\mathrm{QCD}+$ $\mathrm{EW})+\mathrm{NNLL}$ and NLO(QCD + EW) distributions for three central scale choices $\mu_{0}=M / 2$, $\mu_{0}=Q / 2$ and $\mu_{0}=H_{T}$. Only scale uncertainties are shown

Fig. 14 The same as in Fig. 10 but for the $p p \rightarrow t \bar{t} Z$ process

Fig. 15 The same as in Fig. 11 but for the $p p \rightarrow t \bar{t} Z$ process
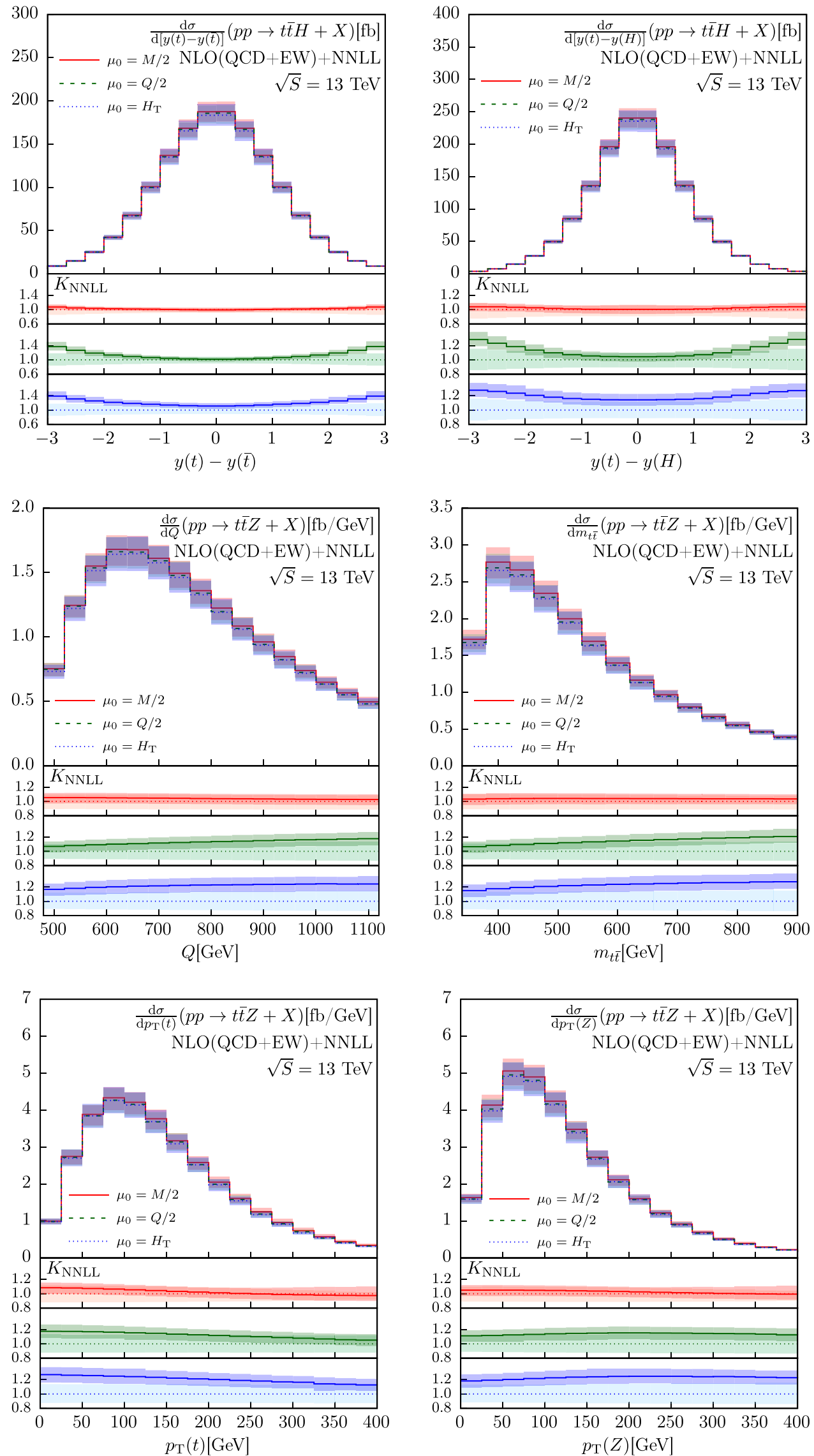
Fig. 16 The same as in Fig. 12 but for the $p p \rightarrow t \bar{t} Z$ process

Fig. 17 The same as in Fig. 13 but for the $p p \rightarrow t \bar{t} Z$ process

Fig. 18 The same as in Fig. 10 but for the $p p \rightarrow t \bar{t} W$ process
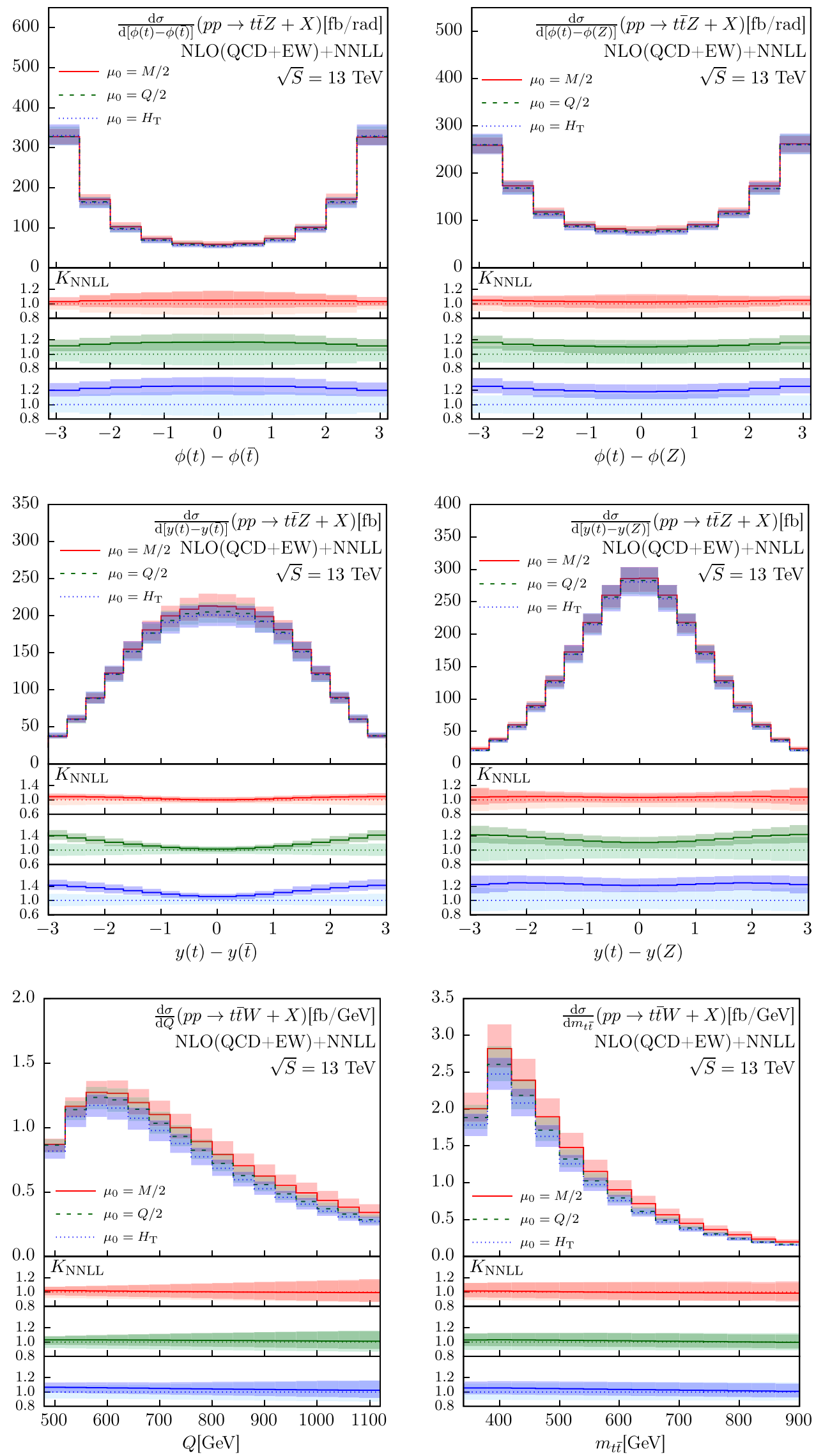
Fig. 19 The same as in Fig. 11 but for the $p p \rightarrow t \bar{t} W$ process

Fig. 20 The same as in Fig. 12 but for the $p p \rightarrow t \bar{t} W$ process

Fig. 21 The same as in Fig. 13 but for the $p p \rightarrow t \bar{t} W$ process
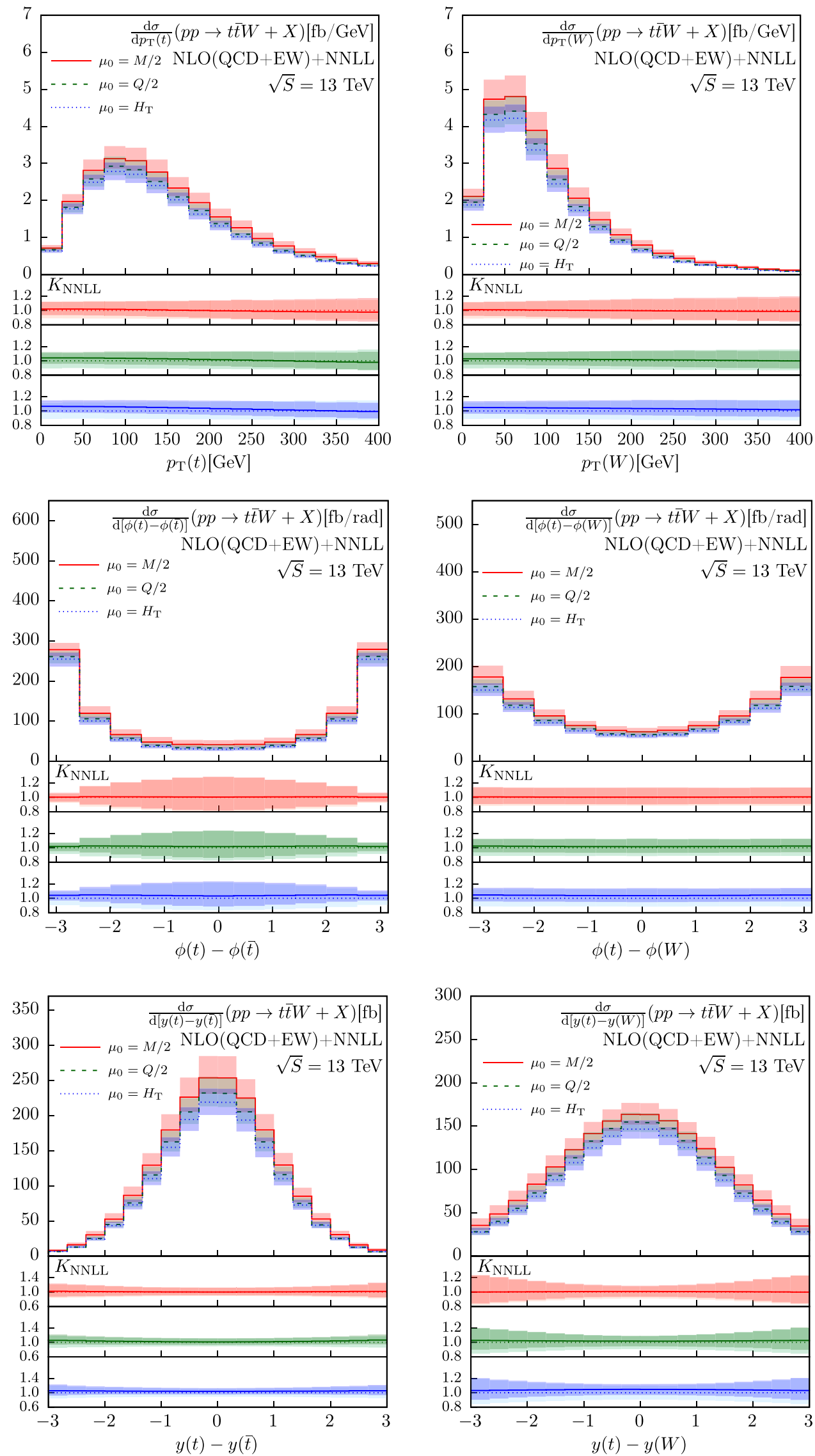
Fig. 22 Comparison of the $p_{T}(Z)$ distribution measured by the CMS collaboration [15] with the NLO (QCD + EW) and $\mathrm{NLO}(\mathrm{QCD}+\mathrm{EW})+\mathrm{NNLL}$ predictions for the central scale choices $\mu_{0}=H_{T}$ and $\mu_{0}=Q / 2$. The shown theoretical uncertainty is from scale variation only

Fig. 23 Comparison of the $p_{T}(Z)$ distribution measured by the CMS collaboration [15] with the NLO $(\mathrm{QCD}+\mathrm{EW})+\mathrm{NNLL}$ predictions for central scale choices considered in this paper (left) and the same comparison for the normalized distribution (right). The shown theoretical uncertainty is from scale variation only
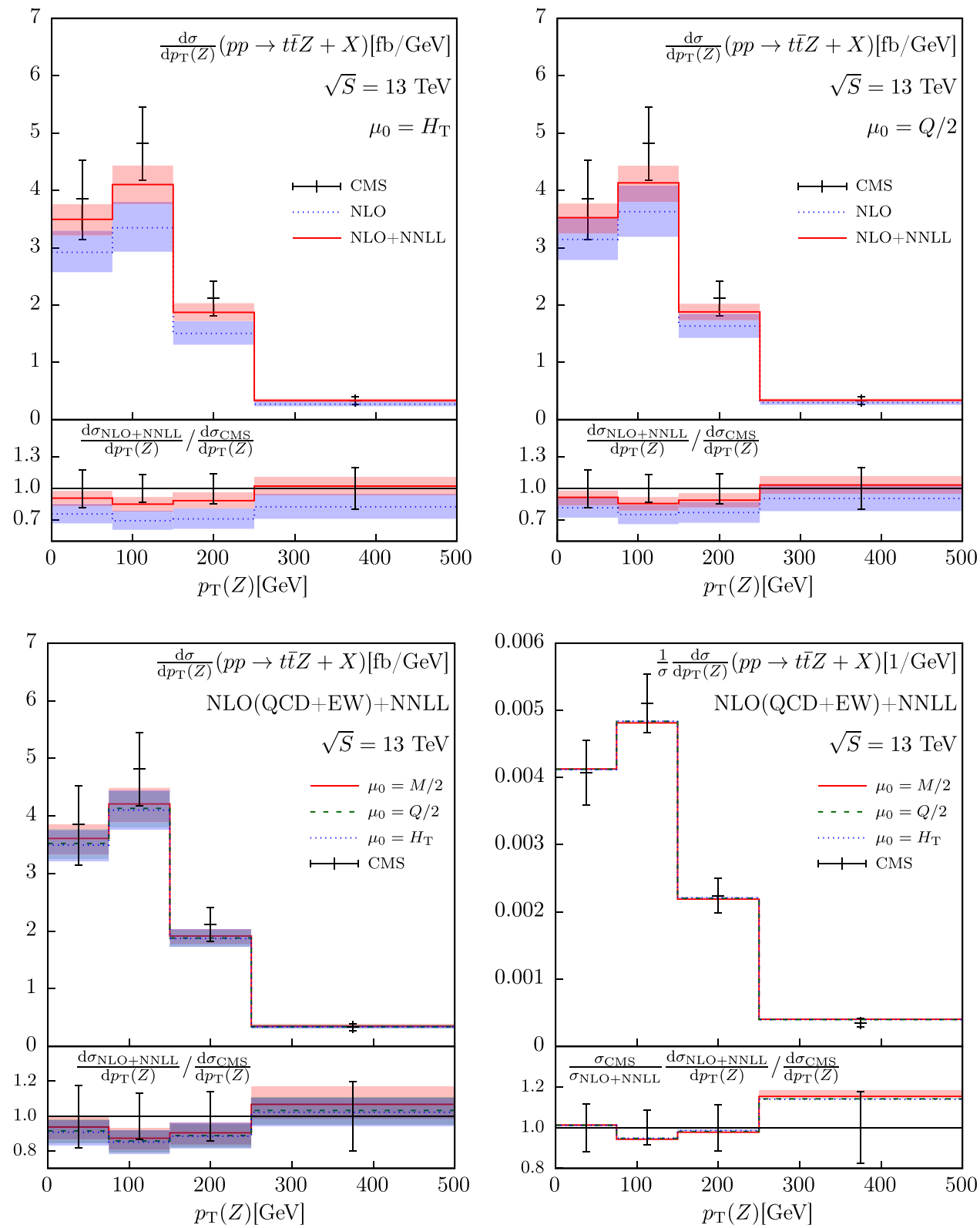

angle $\phi$ and rapidity $y$ differences: $\phi(t)-\phi(\bar{t}), \phi(t)-\phi(B)$, $y(t)-y(\bar{t})$ and $y(t)-y(B)$ for all the considered bosons. The soft gluon resummation effects are found to be significant in the differential cross sections: they affect both the overall normalisation and the shapes. For dynamical scale choices, the magnitude of the NNLL corrections is up to $20-30 \%$ of the $\mathrm{NLO}(\mathrm{QCD}+\mathrm{EW})$ results, but in some kinematic regions the relative NNLL contribution reaches $40 \%$. In general, the estimated theoretical uncertainty of the $\mathrm{NLO}(\mathrm{QCD}+\mathrm{EW})$ + NNLL results is reduced w.r.t. the NLO(QCD + EW) predictions in all distributions. In particular, the resummation greatly reduces dependence on the central scale choice. The strongest improvement of the theoretical accuracy is found for $p p \rightarrow t \bar{t} H, p p \rightarrow t \bar{t} Z$ processes, where two gluon fusion partonic channel is important. The improvement is only moderate for $p p \rightarrow t \bar{t} W^{ \pm}$, where the two gluon channel does not contribute below the NNLO accuracy.

The theoretical estimates are compared to results of the recent CMS measurement [15] of $d \sigma / d p_{T}(Z)$ in $p p \rightarrow t \bar{t} Z$ at $\sqrt{S}=13 \mathrm{TeV}$. The inclusion of soft gluon resummation is shown to significantly improve the agreement between the theoretical predictions and the experimental results, and the theoretical uncertainty due to the central scale choice is nearly completely eliminated in the NLO + NNLL results.

Acknowledgements We are grateful to R. Schoefbeck and J. Knolle for providing us data and very useful exchanges regarding the CMS analysis. This work has been supported by the DFG Grant KU3103/2. AK would like to acknowledge the hospitality of and the support 
from the Erwin Schrödinger International Institute for Mathematics and Physics of the University of Vienna, where part of this work was carried out. The support of the Polish National Science Center (NCN) Grants no. 2017/27/B/ST2/02755 and 2019/32/C/ST2/00202 are gratefully acknowledged. VT acknowledges funding from the European Union's Horizon 2020 research and innovation programme as part of the Marie Skłodowska-Curie Innovative Training Network MCnetITN3 (Grant agreement no. 722104).

Data Availability Statement This manuscript has no associated data or the data will not be deposited. [Authors' comment: The research in this article is purely of a theoretical nature and no experimental data has been collected in the process of working on the paper. The experimental data points to which the theoretical results are compared in Figs. 22 and 23 come from a dedicated publication cited as Ref. [15] in the article.]

Open Access This article is licensed under a Creative Commons Attribution 4.0 International License, which permits use, sharing, adaptation, distribution and reproduction in any medium or format, as long as you give appropriate credit to the original author(s) and the source, provide a link to the Creative Commons licence, and indicate if changes were made. The images or other third party material in this article are included in the article's Creative Commons licence, unless indicated otherwise in a credit line to the material. If material is not included in the article's Creative Commons licence and your intended use is not permitted by statutory regulation or exceeds the permitted use, you will need to obtain permission directly from the copyright holder. To view a copy of this licence, visit http://creativecomm ons.org/licenses/by/4.0/.

Funded by SCOAP ${ }^{3}$.

\section{References}

1. G. Aad et al., ATLAS Collaboration. Phys. Lett. B 740, 222 (2015). arXiv:1409.3122 [hep-ex]

2. G. Aad et al. [ATLAS Collaboration], Eur. Phys. J. C 75 (2015) no.7, 349. arXiv:1503.05066 [hep-ex]

3. V. Khachatryan et al. [CMS Collaboration], JHEP 1409 (2014) 087 [JHEP 1410 (2014) 106]. arXiv:1408.1682 [hep-ex]

4. V. Khachatryan et al. [CMS Collaboration], Eur. Phys. J. C 75 (2015) no.6, 251. arXiv:1502.02485 [hep-ex]

5. G. Aad et al., ATLAS Collaboration. Phys. Lett. B 749, 519 (2015). arXiv:1506.05988 [hep-ex]

6. The ATLAS collaboration [ATLAS Collaboration], ATLASCONF-2016-058, ATLAS-CONF-2016-067, ATLAS-CONF2016-068, ATLAS-CONF-2016-080

7. CMS Collaboration [CMS Collaboration], CMS-PAS-HIG-15008, CMS-PAS-HIG-16-004, CMS-PAS-HIG-16-020, CMS-PASHIG-16-022, CMS-PAS-HIG-16-038, CMS-PAS-HIG-17-004

8. S. Chatrchyan et al., CMS Collaboration. Phys. Rev. Lett. 110, 172002 (2013). arXiv:1303.3239 [hep-ex]

9. V. Khachatryan et al. [CMS Collaboration], Eur. Phys. J. C $\mathbf{7 4}$ (2014) no.9, 3060. arXiv:1406.7830 [hep-ex]

10. G. Aad et al., ATLAS Collaboration. JHEP 1511, 172 (2015). arXiv:1509.05276 [hep-ex]

11. V. Khachatryan et al., CMS Collaboration. JHEP 1601, 096 (2016). arXiv:1510.01131 [hep-ex]

12. M. Aaboud et al. [ATLAS Collaboration], Eur. Phys. J. C 77 (2017) no.1, 40. arXiv:1609.01599 [hep-ex]

13. A.M. Sirunyan et al., CMS Collaboration. JHEP 1808, 011 (2018). arXiv:1711.02547 [hep-ex]

14. The ATLAS collaboration [ATLAS Collaboration], ATLASCONF-2018-047

15. [CMS Collaboration], arXiv:1907.11270 [hep-ex]
16. W. Beenakker, S. Dittmaier, M. Krämer, B. Plumper, M. Spira, P.M. Zerwas, Phys. Rev. Lett. 87, 201805 (2001). arXiv:hep-ph/0107081

17. W. Beenakker, S. Dittmaier, M. Krämer, B. Plumper, M. Spira, P.M. Zerwas, Nucl. Phys. B 653, 151 (2003). arXiv:hep-ph/0211352

18. L. Reina, S. Dawson, Phys. Rev. Lett. 87, 201804 (2001). arXiv:hep-ph/0107101

19. L. Reina, S. Dawson, D. Wackeroth, Phys. Rev. D 65, 053017 (2002). arXiv:hep-ph/0109066

20. S. Dawson, L.H. Orr, L. Reina, D. Wackeroth, Phys. Rev. D 67, 071503 (2003). arXiv:hep-ph/0211438

21. S. Dawson, C. Jackson, L.H. Orr, L. Reina, D. Wackeroth, Phys. Rev. D 68, 034022 (2003). arXiv:hep-ph/0305087

22. A. Lazopoulos, T. McElmurry, K. Melnikov, F. Petriello, Phys. Lett. B 666, 62 (2008). arXiv:0804.2220 [hep-ph]

23. A. Lazopoulos, K. Melnikov, F.J. Petriello, Phys. Rev. D 77, 034021 (2008). arXiv:0709.4044 [hep-ph]

24. A. Kardos, Z. Trocsanyi, C. Papadopoulos, Phys. Rev. D 85, 054015 (2012). arXiv:1111.0610 [hep-ph]

25. F. Maltoni, M.L. Mangano, I. Tsinikos, M. Zaro, Phys. Lett. B 736, 252 (2014). arXiv:1406.3262 [hep-ph]

26. S. Badger, J.M. Campbell, R.K. Ellis, JHEP 1103, 027 (2011). arXiv:1011.6647 [hep-ph]

27. J.M. Campbell, R.K. Ellis, JHEP 1207, 052 (2012). arXiv:1204.5678 [hep-ph]

28. R. Röntsch and M. Schulze, JHEP 1407 (2014) 091 Erratum: [JHEP 1509 (2015) 132] arXiv:1404.1005 [hep-ph]

29. R. Röntsch, M. Schulze, JHEP 1508, 044 (2015). arXiv:1501.05939 [hep-ph]

30. V. Hirschi, R. Frederix, S. Frixione, M.V. Garzelli, F. Maltoni, R. Pittau, JHEP 1105, 044 (2011). arXiv:1103.0621 [hep-ph]

31. J. Alwall et al., JHEP 1407, 079 (2014). arXiv:1405.0301 [hep-ph]

32. R. Frederix, S. Frixione, V. Hirschi, F. Maltoni, R. Pittau, P. Torrielli, Phys. Lett. B 701, 427 (2011). arXiv:1104.5613 [hep-ph]

33. M.V. Garzelli, A. Kardos, C.G. Papadopoulos, Z. Trocsanyi, Europhys. Lett. 96, 11001 (2011). arXiv:1108.0387 [hep-ph]

34. H. B. Hartanto, B. Jager, L. Reina, D. Wackeroth, Phys. Rev. D 91 (2015) 9, 094003. arXiv:1501.04498 [hep-ph]

35. M.V. Garzelli, A. Kardos, C.G. Papadopoulos, Z. Trocsanyi, JHEP 1211, 056 (2012). arXiv:1208.2665 [hep-ph]

36. F. Maltoni, D. Pagani, I. Tsinikos, JHEP 1602, 113 (2016). arXiv:1507.05640 [hep-ph]

37. S. Frixione, V. Hirschi, D. Pagani, H.-S. Shao, M. Zaro, JHEP 1506, 184 (2015). arXiv:1504.03446 [hep-ph]

38. S. Frixione, V. Hirschi, D. Pagani, H.S. Shao, M. Zaro, JHEP 1409, 065 (2014). arXiv:1407.0823 [hep-ph]

39. R. Frederix, D. Pagani, M. Zaro, JHEP 1802, 031 (2018). arXiv:1711.02116 [hep-ph]

40. Y. Zhang, W.G. Ma, R.Y. Zhang, C. Chen, L. Guo, Phys. Lett. B 738, 1 (2014). arXiv:1407.1110 [hep-ph]

41. G. Bevilacqua, H.B. Hartanto, M. Kraus, T. Weber, M. Worek, JHEP 1911, 001 (2019). arXiv:1907.09359 [hep-ph]

42. A. Denner, R. Feger, JHEP 1511, 209 (2015). arXiv:1506.07448 [hep-ph]

43. A. Denner, J.N. Lang, M. Pellen, S. Uccirati, JHEP 1702, 053 (2017). arXiv:1612.07138 [hep-ph]

44. G.F. Sterman, Nucl. Phys. B 281, 310 (1987)

45. S. Catani, L. Trentadue, Nucl. Phys. B 327, 323 (1989)

46. C.W. Bauer, S. Fleming, D. Pirjol, I.W. Stewart, Phys. Rev. D 63, 114020 (2001). arXiv:hep-ph/0011336

47. C.W. Bauer, D. Pirjol, I.W. Stewart, Phys. Rev. D 65, 054022 (2002). arXiv:hep-ph/0109045

48. T. Becher, M. Neubert, Phys. Rev. Lett. 97, 082001 (2006). arXiv:hep-ph/0605050

49. M. Beneke, A.P. Chapovsky, M. Diehl, T. Feldmann, Nucl. Phys. B 643, 431 (2002). arXiv:hep-ph/0206152 
50. A. Kulesza, L. Motyka, T. Stebel, V. Theeuwes, JHEP 1603, 065 (2016). arXiv:1509.02780 [hep-ph]

51. A. Kulesza, L. Motyka, T. Stebel, V. Theeuwes, PoS LHCP 2016, 084 (2016). arXiv:1609.01619 [hep-ph]

52. A. Kulesza, L. Motyka, T. Stebel, V. Theeuwes, Phys. Rev. D 97 (2018) no.11, 114007. arXiv:1704.03363 [hep-ph]

53. A. Kulesza, L. Motyka, T. Stebel, V. Theeuwes, PoS EPS HEP2017 (2017) 339. arXiv:1710.06358 [hep-ph]

54. A. Kulesza, L. Motyka, D. Schwartländer, T. Stebel, V. Theeuwes, PoS EPS -HEP2017 (2017) 465. arXiv:1710.06810 [hep-ph]

55. A. Kulesza, L. Motyka, D. Schwartländer, T. Stebel, V. Theeuwes, Eur. Phys. J. C 79 (2019) no.3, 249. arXiv:1812.08622 [hep-ph]

56. A. Kulesza, L. Motyka, D. Schwartländer, T. Stebel, V. Theeuwes, PoS CORFU 2018, 024 (2019)

57. A. Kulesza, L. Motyka, D. Schwartländer, T. Stebel, V. Theeuwes. arXiv:1905.07815 [hep-ph]

58. A. Broggio, A. Ferroglia, B.D. Pecjak, A. Signer, L.L. Yang, JHEP 1603, 124 (2016). arXiv:1510.01914 [hep-ph]

59. A. Broggio, A. Ferroglia, B.D. Pecjak, L.L. Yang, JHEP 1702, 126 (2017). arXiv:1611.00049 [hep-ph]

60. A. Broggio, A. Ferroglia, G. Ossola, B.D. Pecjak, JHEP 1609, 089 (2016). arXiv:1607.05303 [hep-ph]

61. A. Broggio, A. Ferroglia, G. Ossola, B.D. Pecjak, R.D. Sameshima, JHEP 1704, 105 (2017). arXiv:1702.00800 [hep-ph]

62. A. Broggio, A. Ferroglia, R. Frederix, D. Pagani, B.D. Pecjak, I. Tsinikos, JHEP 1908, 039 (2019). arXiv:1907.04343 [hep-ph]

63. H. T. Li, C. S. Li, S. A. Li, Phys. Rev. D 90 (2014) 9, 094009. arXiv:1409.1460 [hep-ph]

64. W.L. Ju, L.L. Yang, JHEP 1906, 050 (2019). arXiv:1904.08744 [hep-ph]
65. S. Catani, M.L. Mangano, P. Nason, L. Trentadue, Nucl. Phys. B 478, 273 (1996). arXiv:hep-ph/9604351

66. R. Frederix, S. Frixione, V. Hirschi, D. Pagani, H.-S. Shao, M. Zaro, JHEP 1807, 185 (2018). arXiv:1804.10017 [hep-ph]

67. E. Laenen, G. Oderda, G.F. Sterman, Phys. Lett. B 438, 173 (1998). arXiv:hep-ph/9806467

68. A. Denner, S. Dittmaier, M.Grazzini, R. Harlander, R. Thorne, M. Spira, M. Steinhauser, LHCHXSWG-INT-2015-006

69. S. Dittmaier et al. [LHC Higgs Cross Section Working Group Collaboration]. arXiv:1101.0593 [hep-ph]

70. J. Butterworth et al., J. Phys. G 43, 023001 (2016). arXiv: 1510.03865 [hep-ph]

71. S. Dulat et al., Phys. Rev. D 93 (2016) no.3, 033006. arXiv:1506.07443 [hep-ph]

72. L. A. Harland-Lang, A. D. Martin, P. Motylinski, R. S. Thorne, Eur. Phys. J. C 75 (2015) no.5, 204. arXiv:1412.3989 [hep-ph]

73. R.D. Ball et al., NNPDF Collaboration. JHEP 1504, 040 (2015). arXiv: 1410.8849 [hep-ph]

74. J. Gao, P. Nadolsky, JHEP 1407, 035 (2014). arXiv:1401.0013 [hep-ph]

75. S. Carrazza, S. Forte, Z. Kassabov, J. I. Latorre, J. Rojo, Eur. Phys. J. C 75 (2015) no.8, 369. arXiv:1505.06736 [hep-ph]

76. A. Manohar, P. Nason, G. P. Salam, G. Zanderighi, Phys. Rev. Lett. 117 (2016) no.24, 242002. arXiv:1607.04266 [hep-ph]

77. A.V. Manohar, P. Nason, G.P. Salam, G. Zanderighi, JHEP 1712, 046 (2017). arXiv:1708.01256 [hep-ph]

78. J. A. M. Vermaseren. arXiv:math-ph/0010025

79. T. van Ritbergen, A.N. Schellekens, J.A.M. Vermaseren, Int. J. Mod. Phys. A 14, 41 (1999). arXiv:hep-ph/9802376 